Portland State University

PDXScholar

5-8-1997

\title{
Molecular Fluorescence at a Rough Surface: The Orientation Effects
}

William Lee Blacke

Portland State University

Follow this and additional works at: https://pdxscholar.library.pdx.edu/open_access_etds

Part of the Physics Commons

Let us know how access to this document benefits you.

\section{Recommended Citation}

Blacke, William Lee, "Molecular Fluorescence at a Rough Surface: The Orientation Effects" (1997).

Dissertations and Theses. Paper 5820.

https://doi.org/10.15760/etd.7691

This Thesis is brought to you for free and open access. It has been accepted for inclusion in Dissertations and Theses by an authorized administrator of PDXScholar. Please contact us if we can make this document more accessible: pdxscholar@pdx.edu. 


\section{THESIS APPROVAL}

The abstract and thesis of William Lee Blacke for the Master of

Science in Physics were presented May 8, 1997, and accepted by the thesis committee and department.

COMMITTEE APPROVALS:

DEPARTMENTAL APPROVAL:

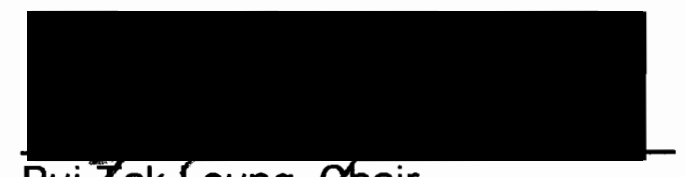

Pui-̄̄ak Leung, Chair

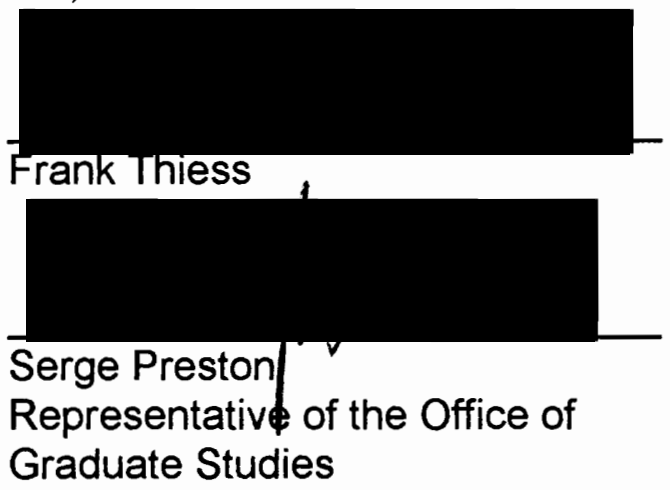

Graduate Studies

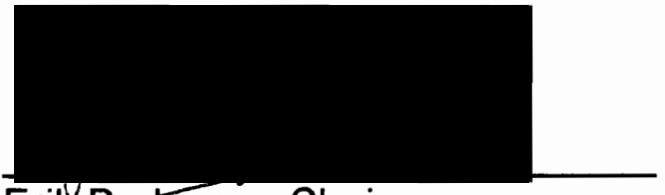

Erik Bodegom, Chair

Department of Physics

ACCEPTED FOR PORTLAND STATE UNIVERSITY BY THE LIBRARY by

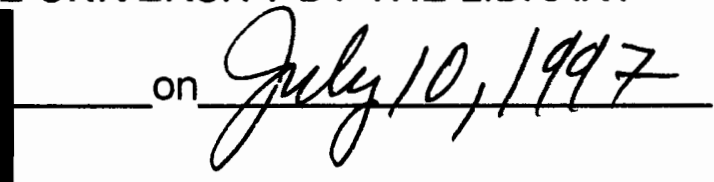




\section{ABSTRACT}

An abstract of the thesis of William Lee Blacke for the Master of Science in Physics presented May 20, 1997

Title: Molecular Fluorescence at a Rough Surface: the Orientation Effects.

The interaction between an emitting molecular dipole and a conducting substrate with a periodic surface roughness is looked at with particular interest in the different orientations of the dipole with respect to the substrate surface. A previous dynamical, perturbative theory for the effects of perpendicular dipole is extended to treat a dipole oriented parallel to the surface of the substrate. The results are then applied to study the modified fluorescence characteristics of the emitting dipoles. Numerical results demonstrate that some fluorescence characteristics are extremely sensitive to the molecular orientation with the dipole oriented along the grating ( $x$ direction) exhibiting unique behavior. One 
possible consequence of the interaction is the lengthening of molecular fluorescence lifetimes by manipulating the parameters of the system. Also, the usual step for averaging the orientations for experimental values is scrutinized. 
MOLECULAR FLUORESCENCE AT A ROUGH SURFACE :

THE ORIENTATION EFFECTS

by

WILLIAM LEE BLACKE

A thesis submitted in partial fulfillment of the requirements for the degree of

MASTER OF SCIENCE

in

PHYSICS

Portland State University

1997 
[blank ] 
[blank] 


\section{Table of Contents}

List of Figures....................... Chapter

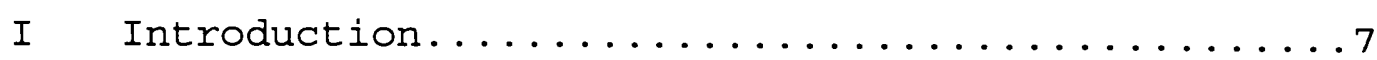

II Motivation for Study of Parallel Dipole......12

III Dynamical Perturbation Theory.............15

IV Molecular Fluorescence Characteristics.......22

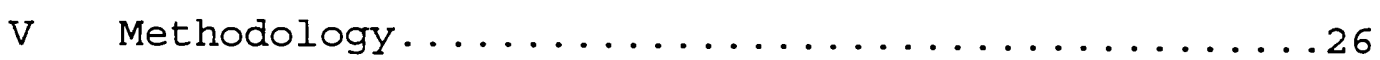

VI Numerical Results and Discussion.........27

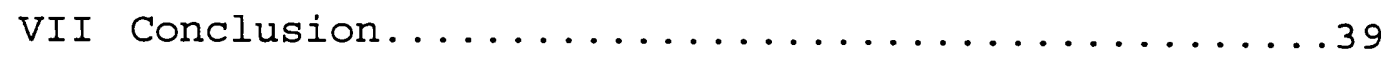

References......................41

Appendices.....................43 


\section{FIGURE CAPTIONS}

1. Accurate prediction for the far distance case from the CPS theory and the discrepancy in experimental results for close dipole distances. . . . . . . . . . . . . . pg.10

2. The geometry of the dipole-substrate system. The dipole orientations and distance are shown, along with the dielectric constants and roughness parameters of the substrate. . . . . . . . . . . . . . . . . . pg.13

3. Comparison between the static and dynamic theories by plotting Im $G^{R}$ in Eqs. $(38-40)$ and in Eqs. $(42-44)$ as a function of the dipole-substrate distance. The labels $x$, $y$, and $z$ indicate the three orientations of the molecule. . . . . . . . . . . . . . . . pg.28

4. Ratio of the decay rate at rough surface to that at flat surface as a function of distance, plotted for three different emission frequencies. . . . . . . . . pg.30

5. Similar to Fig. 4, except the surface-induced decay rate is normalized to the free molecule value, rather than to the flat surface value. The case for a flat surface and that for a grating with $Q=0.02 \mathrm{~nm}^{-1}$ are shown for the emission frequency at $2.5 \mathrm{eV}$. . . . . . . . . pg.32 
6. Normalized decay rate spectrum for a grating surface with $Q=0.02 \mathrm{~nm}^{-1}$ as a function of emission frequency at three different molecule-surface distances. . . . pg.35

7. Normalized molecular Erequency-shifts for a grating surface with $Q=0.02 \mathrm{~nm}^{-1}$ as a function of distance for three different emission frequencies. . . . . . . pg.36

8. Same as in Fig. 7, except for a plot as a function of emission frequencies at a fixed distance of $10 \mathrm{~nm}$. The results are shown for a flat, a grating surface with $\mathrm{Q}=0.02 \mathrm{~nm}^{-1}$, and one with $\mathrm{Q}=0.05 \mathrm{~nm}^{-1}$, respectively. . pg.38 


\section{INTRODUCTION}

The interaction of a molecular dipole and a conducting surface has been studied in great detail since the early 70's[1-3]. In particular, the study of the fluorescence of molecules (e.g. dyes) in the vicinity of a conducting surface has held a great interest.

Although there have been many theories, both classical and quantum mechanical, an efficient, classical theory for this phenomenon was formulated by Chance, Prock, and Silbey (CPS) [3]. This theory has successfully accounted for many of the far distance experimental results. In the CPS theory, the fluorescing molecule is modeled as an emitting point dipole with its emission characteristics modified by the field ( $\left.E^{r}\right)$ reflected from the substrate surface to the dipole position. This method is analogous to an earlier approach by sommerfeld in calculating the modified characteristics of an antenna near the Earth's surface[4]. In our problem, the antenna is replaced by the molecular dipole and the Earth's surface is replaced by the conducting substrate. The full electrodynamics are then solved with the application of the Sommerfeld theory. This theory models the molecule as an oscillating electric dipole. The equations of motion for an oscillating dipole can be found by starting with Newton's Second Law and including the forces inherent in the system and the 
external forces acting on the system. The internal force in the system includes a harmonic force acting on the on the bound electron oscillating with a frequency $\omega$ as well as the intrinsic damping force due to the fluctuation in vacuum. When the dipole is near a metal substrate, as is the case for the present work, there is an external force on the dipole from the electric field reflected from the substrate surface. A sum of these forces results in a differential equation of motion in terms of the mass, electric field, and position coordinate. The solution to this differential equation should be oscillatory in nature.

The damping force indicates an exponential decay in the solution which introduces two important quantities in terms of the reflected field at the dipole position. These are $\Delta \omega$, the frequency shift, and $\gamma$, the decay for the oscillating dipole. Further manipulation of the solution leads to expressions for these characteristics as follows [3] :

$$
\begin{aligned}
& \frac{\Delta \omega}{\gamma_{0}}=\frac{-3 q}{4 \mu \mathrm{k}^{3}} \operatorname{Re} E^{r} \\
& \frac{\gamma}{\gamma_{0}}=1+\frac{3 q}{2 \mu \mathrm{k}^{3}} \operatorname{Im} \mathrm{E}^{\mathrm{r}}
\end{aligned}
$$

where $\mu$ is the dipole moment, $q$ the intrinsic quantum yield, and $k$ the emission wave number of the admolecule. 
Note that strictly speaking the CPS theory is limited to a surface of perfect flatness which can be a reasonable idealization in actual experiments for molecules at large distances from a well-prepared surface. It was the observation in the 80's of deviations from the CPS theory at close molecule-surface distances (d<10nm [5]), that led to a series of studies on the corrections to the CPS theory due to surface roughness $[6-10]$. Figure 1 shows the accurate predictions for the CPS theory for the fardistance case and the discrepancy for the near-distance case.

Both the static (image) [6-7] and the more exact dynamic [8-10] theories have been considered for both random [6] and periodic [7-10] roughness in the literature. For perpendicularly-oriented molecular dipoles, the previous works have indicated that surface roughness can either enhance or suppress the effects from a flat surface, and can lead to extra morphologicallyinduced resonance's in the decay rate spectrum of the admolecules.

It is the purpose of this thesis to enlarge the previous dynamical theory [8] for the interaction of an emitting dipole with a rough surface which will be modeled as a grating. The previous theory primarily studied the effects of a dipole oriented perpendicular to the surface. The present work will include cases with a parallel dipole 

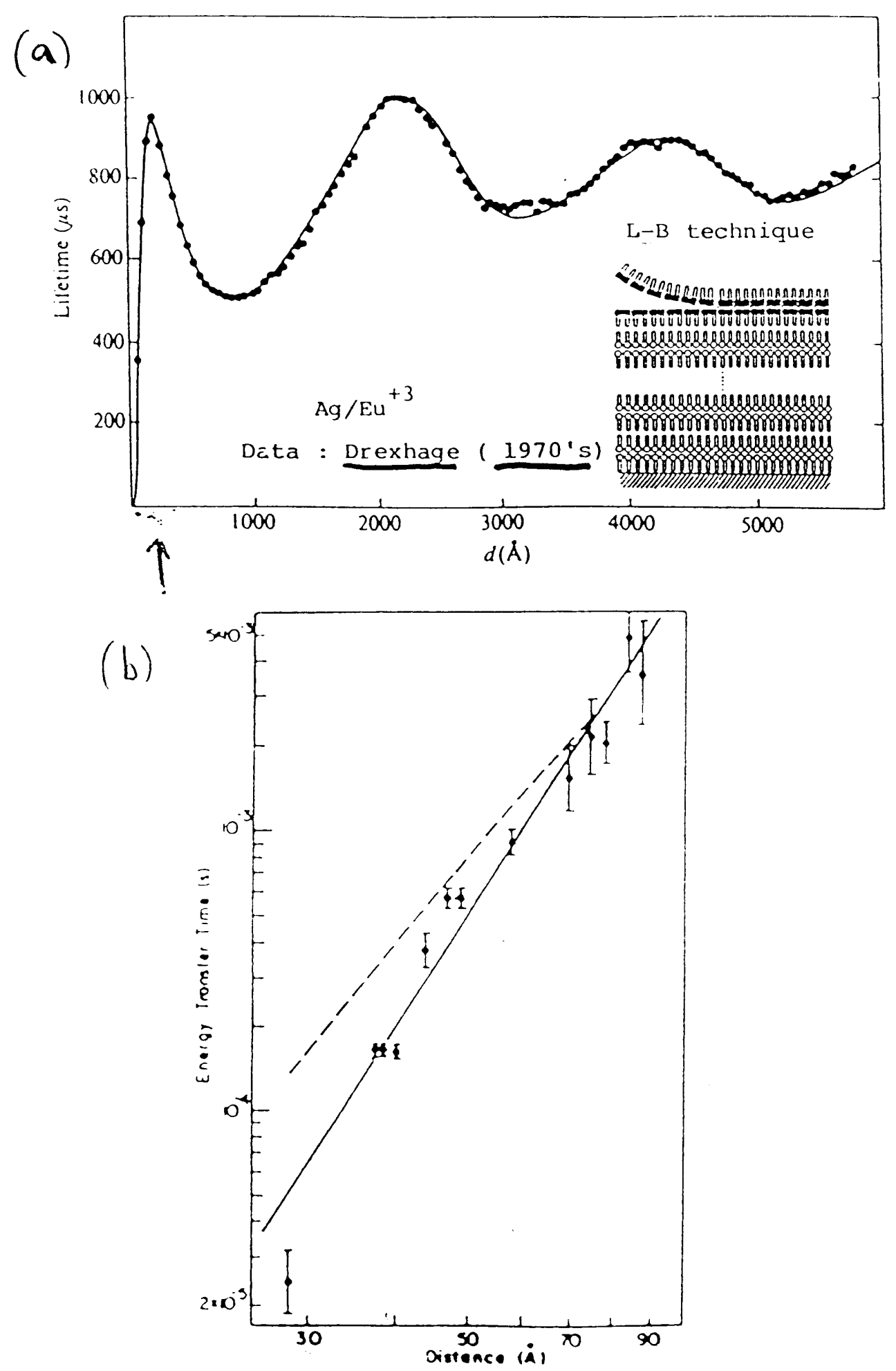

Fig. 1. (a) Results from CPS theory for the lifetime as a function of dipole distance[3]. (b) Divergence of experimental data from the CPS theory for near-distance case[5]. 
orientation in the $\mathrm{x}$ and $\mathrm{y}$ directions as well. The results will be applied to study the effects on the modified fluorescence characteristics due to the different orientations of the admolecules. Among other implications from the results, the practice of "averaging the molecular orientations" for a randomly oriented ensemble of admolecules is questioned in the case of patterned substrate surfaces.

This thesis will be organized as follows. Chapter II will address the motivation for the current study. In Chapter III, a summary and continuation of the previous perturbative method for calculating the reflected field $\left(E^{r}\right)$ from a rough interface will be shown. The resulting modified fluorescence characteristics for both the perpendicular and parallel dipoles and a comparison with those from the static theory will be given in Chapter IV. Chapter $\mathrm{V}$ discusses the methodology in obtaining the numerical results for a silver grating substrate found in Chapter VI with a conclusion in Chapter VII. 


\section{MOTIVATION FOR STUDY OF PARALLEL DIPOLE}

The problem involves the dynamical interaction between an oscillating point dipole (in vacuum) with a semiinfinite substrate grating surface as depicted in Fig. 2. For the case with a dipole moment $(\vec{\mu})$ perpendicular (along z) to the substrate surface, the problem has been solved previously in a perturbative approach in both a dynamic [8] and a simpler static [7] approach via the application of the image theory (IT). For the case of an arbitrarily oriented dipole, only IT has been carried out in the previous investigations by deriving results for the two cases with parallel oriented dipoles (along $x$ and $y$, respectively). It is well established that IT is accurate only when (1) the molecular dipole is located at a distance (d) much smaller than its emission wavelength, (2) the substrate conductivity is not too high so that d $>\delta$, the skin depth of the metal substrate, and (3) the emission frequency is not close to the morphologicallyinduced resonance frequency for resonant radiative energy transfer to take place [11]. Hence, to obtain a model which can study the orientation effects more accurately, this thesis will generalize the previous dynamical theory [8] to cases with molecular orientations parallel to the plane of the substrate surface. As is clear from Eqs. (I) and (2), the main quantity which needs to be calculated in 


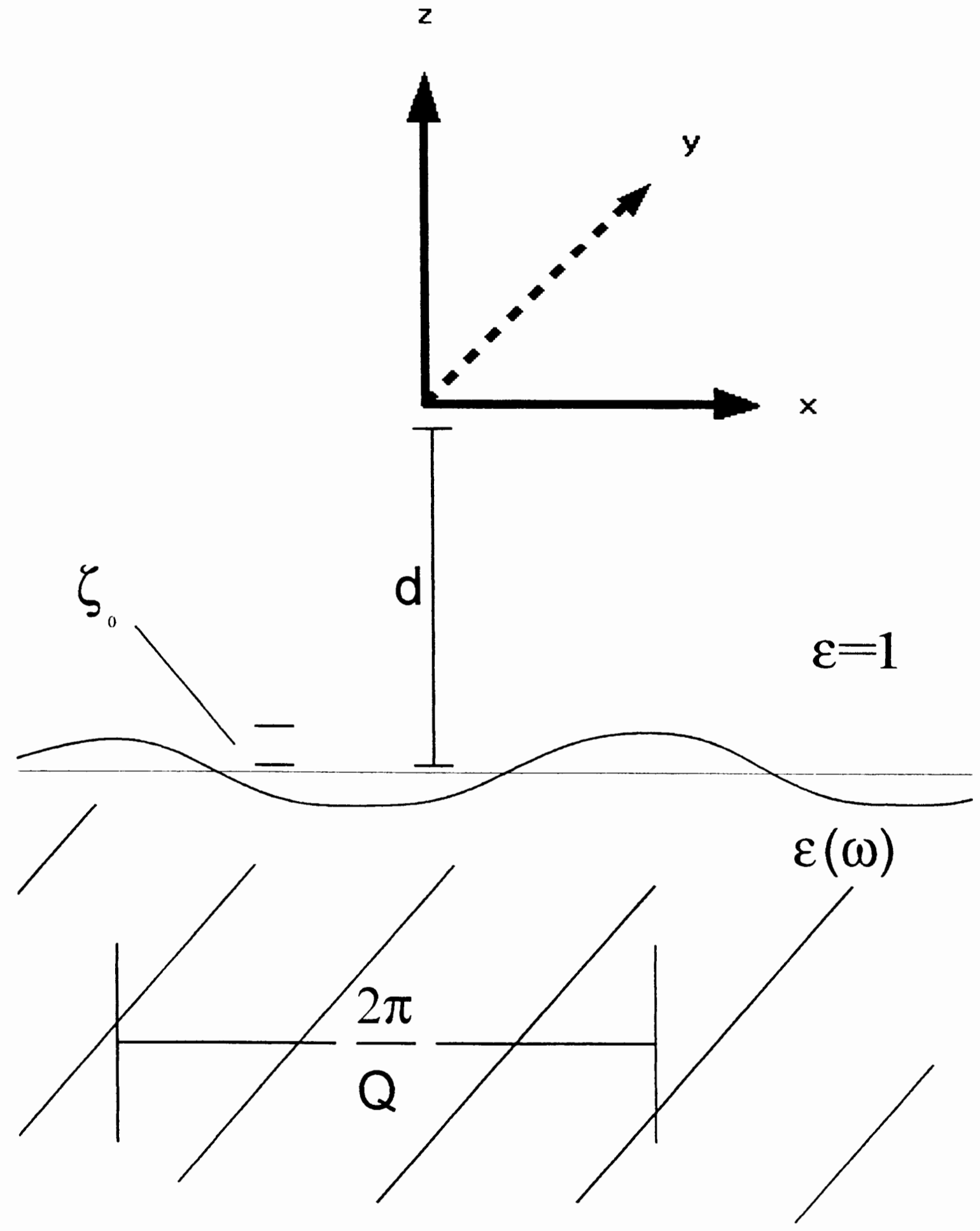

Fig. 2 The geometry of the dipole substrate system. The dipole orientations and distance are shown, along with the dielectric constants and roughness parameters of the substrate. 
order to determine the modified emission characteristics for the admolecules is the reflected field from the rough substrate surface acting at the dipole site. To solve the electrodynamic (Maxwell) equations in a perturbative approach with the roughness as a perturbation parameter, we will follow the original Green-function formulation of Maradudin and Mills (MM) [12] with modifications from the work of Agarwal [13]. The MM theory as applied to this dipole - substrate problem will need to be reviewed and clarified in Chapter III before the complete set of expressions for the fluorescence characteristics in Chapter IV can be presented. The theory will be limited to first order perturbation and hence the degree of roughness is assumed to be small throughout the present work. 


\section{A DYNAMICAL PERTURBATION THEORY}

According to the MM theory [12], the surface roughness of the conducting substrate can be regarded as a source to the homogeneous Helmholtz equation for the case of a perfectly flat interface. The dielectric constant of the surface-medium system can be written as:

$$
\varepsilon(\mathrm{z} ; \omega)=\Theta(\mathrm{z}-\zeta(\mathrm{x}, \mathrm{y}))+\varepsilon(\omega) \Theta(\zeta(\mathrm{x}, \mathrm{y})-\mathrm{z}) \quad,
$$

where $\Theta(z)$ is the Heavyside step function and $\zeta$ is the profile function for the roughness. Upon an expansion of Eq. 3 in powers of $\zeta(x, y)$, we obtain

$$
\varepsilon(\mathrm{z} ; \omega)=\varepsilon_{0}(\mathrm{z} ; \omega)+[\varepsilon(\omega)-1] \zeta(\mathrm{x}, \mathrm{y}) \delta(\mathrm{z})+\mathrm{O}\left(\zeta^{2}\right),
$$

where

$$
\varepsilon_{0}(z ; \omega)= \begin{cases}1, & z>0 \\ \varepsilon(\omega), & z<0 .\end{cases}
$$

This gives the desired values for the dielectric constant for the two media.

To compute the electric field reflected from this rough surface, we start with Maxwell's equation,

$$
\nabla \times \nabla \times \overrightarrow{\mathrm{E}}=-\frac{\omega^{2}}{\mathrm{k}^{2}} \frac{\partial^{2}}{\partial \mathrm{t}^{2}} \overrightarrow{\mathrm{D}}
$$

and assume the following solution,

$$
\begin{aligned}
& \overrightarrow{\mathrm{E}}(\overrightarrow{\mathrm{r}} ; \mathrm{t})=\overrightarrow{\mathrm{E}}(\overrightarrow{\mathrm{r}} ; \omega) \mathrm{e}^{-\mathrm{i} \omega t} \\
& \overrightarrow{\mathrm{D}}(\overrightarrow{\mathrm{r}} ; \mathrm{t})=\overrightarrow{\mathrm{D}}(\overrightarrow{\mathrm{r}} ; \omega) \mathrm{e}^{-\mathrm{i} \omega t}
\end{aligned}
$$

Using, 


$$
\overrightarrow{\mathrm{D}}(\overrightarrow{\mathrm{r}} ; \omega)=\varepsilon(z ; \omega) \overrightarrow{\mathrm{E}}(\overrightarrow{\mathrm{r}} ; \omega),
$$

one can find the Fourier coefficient of the electric field $\overrightarrow{\mathrm{E}}(\overrightarrow{\mathrm{r}} ; \omega)$ satisfying:

$$
\nabla \times \nabla \times \overrightarrow{\mathrm{E}}(\overrightarrow{\mathrm{r}} ; \omega)-\varepsilon_{0}(\mathrm{z} ; \omega) \mathrm{k}^{2} \overrightarrow{\mathrm{E}}(\overrightarrow{\mathrm{r}} ; \omega)=\mathrm{k}^{2}[\varepsilon(\omega)-1] \zeta(\mathrm{x}, \mathrm{y}) \delta(\mathrm{z}) \overrightarrow{\mathrm{E}}(\overrightarrow{\mathrm{r}} ; \omega) .
$$

To implement the Born-type approximation, it is desirable to transform the partial differential equation into an integral equation. To do this, we must introduce the Green's function, $\mathrm{D}_{\mu v}\left(\overrightarrow{\mathrm{r}}, \overrightarrow{\mathrm{r}}^{\prime} ; \omega\right)$, as the solution to the equation:

$$
\left(\varepsilon_{0}(z ; \omega) k^{2} \delta_{\lambda \mu}-\frac{\partial^{2}}{\partial x_{\lambda} \partial x_{\mu}}+\delta_{\lambda \mu} \nabla^{2}\right) D_{\mu v}\left(\vec{r}, \vec{r}^{\prime} ; \omega\right)=4 \pi \delta_{\lambda \nu} \delta\left(\vec{r}-\vec{r}^{\prime}\right)
$$

The field can hence be expressed in terms of $D_{\mu v}$ as:

$$
E_{\mu}(\vec{r}, \omega)=E_{\mu}^{0}(\vec{r}, \omega)-\frac{k^{2}}{4 \pi}[\varepsilon(\omega)-1] \int d^{3} r^{\prime} D_{\mu v}\left(\vec{r}, \vec{r}^{\prime} ; \omega\right) \zeta\left(x^{\prime}, y^{\prime}\right) \delta(z) E_{v}\left(\vec{r}^{\prime} ; \omega\right),
$$

where $E_{\mu}^{0}$ is the total field for the case with a flat interface. It should be noted that the Einstein summation convention of summing over the repeated indices, is used throughout the present work.

Furthermore, it will be convenient to represent the following functions by their 2D Fourier transforms as follows :

$$
D_{\mu \nu}\left(\vec{r}, \vec{r}^{\prime} ; \omega\right)=\int \frac{d^{2} \vec{k}_{\|}}{4 \pi^{2}} e^{i \vec{k}_{\|}\left(\vec{r}_{i}-\vec{r}_{i \|}\right)} d_{\mu \nu}\left(\vec{k}_{\|}, \omega \mid z z^{\prime}\right),
$$




$$
\begin{aligned}
& \zeta\left(\vec{r}_{\|}\right)=\int \frac{d^{2} \vec{k}_{\|}}{4 \pi^{2}} e^{i \vec{k}_{\|} \cdot \vec{r}} \hat{\zeta}\left(\vec{k}_{\|}\right) \\
& E_{\mu}^{(0)}(\vec{r}, \omega)=e^{i \vec{k}_{\|}^{(0)} \cdot \vec{r}_{1}} E_{\mu}^{(0)}\left(\vec{k}_{\|}^{(0)}, \omega \mid z\right)
\end{aligned}
$$

with the two dimensional vectors $\vec{k}_{y}=\left(k_{x}, k_{y}, 0\right)$ and $\vec{r}_{l}=$ $(x, y, 0)$ where the || denotes an orientation parallel to the surface. The Fourier transformed Green dyadic function, $d_{\mu v}$, can be obtained from the original MM paper[12] (see Appendix B). Substituting these back into Eq.11 we obtain the $\mu$ th component of the reflected field due to the roughness in the following form:

$$
\mathrm{E}_{\mu}^{\mathrm{rR}}=-\frac{\mathrm{k}^{2}}{16 \pi^{3}}(\varepsilon-1) \int \mathrm{d}^{2} \overrightarrow{\mathrm{k}}_{\|} \mathrm{e}^{\mathrm{i} \overrightarrow{\mathrm{k}}_{1} \cdot \overrightarrow{\mathrm{r}}_{\|}} \hat{\zeta}\left(\overrightarrow{\mathrm{k}}_{\|}-\overrightarrow{\mathrm{k}}_{\|}^{(0)}\right) \int \mathrm{d} \mathrm{z}^{\prime} \mathrm{d}_{\mu v}\left(\overrightarrow{\mathrm{k}}_{\|}, \omega \mid z z^{\prime}\right) \delta\left(\mathrm{z}^{\prime}\right) \mathrm{E}_{v}^{(0)}\left(\overrightarrow{\mathrm{k}}_{\|}^{(0)}, \omega \mid \mathrm{z}^{\prime}\right)
$$

Strictly speaking, Eq.(15) is valid only for the case of a plane incident wave with a constant $\overrightarrow{\mathrm{k}}_{\|}^{(0)}$ vector, so that $\mathrm{E}^{(0)}\left(\overrightarrow{\mathrm{k}}_{\|}^{(0)}, \omega \mid \mathrm{z}^{\prime}\right)=\mathrm{e}^{\mathrm{i} \overrightarrow{\mathrm{k}}_{1}^{(0)} \cdot \overrightarrow{\mathrm{r}}} \mathrm{E}^{(0)}\left(\omega \mid \mathrm{z}^{\prime}\right)$. For a dipole emission as the incident source, a full 2D Fourier transform has to be applied for $\mathrm{E}^{(0)}$ to sum over all possible $\overrightarrow{\mathrm{k}}_{\|}^{(0)}$ and Eq. (15) must take the following form :

$\mathrm{E}_{\mu}^{\mathrm{rR}}=-\frac{\mathrm{k}^{2}}{16 \pi^{3}}(\varepsilon-1) \int \mathrm{d}^{2} \overrightarrow{\mathrm{k}}_{\|}^{(0)} \int \mathrm{d}^{2} \overrightarrow{\mathrm{k}}_{\|} \mathrm{e}^{i \overrightarrow{\mathrm{k}}_{\|} \cdot \overrightarrow{\mathrm{t}}_{\|}} \hat{\zeta}\left(\overrightarrow{\mathrm{k}}_{\|}-\overrightarrow{\mathrm{k}}_{\|}^{(0)}\right) \int \mathrm{d} \mathrm{z}^{\prime} \mathrm{d}_{\mu \nu}\left(\overrightarrow{\mathrm{k}}_{\|}, \omega \mid z z^{\prime}\right) \delta\left(\mathrm{z}^{\prime}\right) \mathrm{E}_{v}^{(0)}\left(\overrightarrow{\mathrm{k}}_{\|}^{(0)}, \omega \mid \mathrm{z}^{\prime}\right)$ 
However, since we shall work only in the limit of shallow roughness, we shall further assume that only incident waves with $\overrightarrow{\mathrm{k}}_{\|}^{(0)} \approx 0$ will be reflected to the dipole site. Hence we shall approximate $\mathrm{E}_{\mathrm{v}}^{(0)}$ in Eq. (16) in the form : $\mathrm{E}_{v}^{(0)}\left(\overrightarrow{\mathrm{k}}_{\|}^{(0)}, \omega \mid \mathrm{z}^{\prime}\right)=\mathrm{E}_{v}^{(0)}\left(\omega \mid \mathrm{z}^{\prime}\right) \delta\left(\overrightarrow{\mathrm{k}}_{\|}^{(0)}\right)$, and by having the dipole located at $\vec{r}=(0,0, d)$, we finally obtain the roughness contribution to the "parallel components" of the reflected field for a point on the $z$-axis given by :

$$
\begin{array}{r}
E_{x}^{r R}(z, \omega)=-\frac{k^{2}}{16 \pi^{3}}[\varepsilon(\omega)-1] \int d^{2} \vec{k}_{\|} \hat{\zeta}\left(\vec{k}_{\|}\right) \int d z^{\prime}\left[d_{x x} E_{x}^{(0)}+d_{x y} E_{y}^{(0)}+d_{x z} E_{z}^{(0)}\right] \delta\left(z^{\prime}\right) \\
E_{y}^{r R}(z, \omega)=-\frac{k^{2}}{16 \pi^{3}}[\varepsilon(\omega)-1] \int d^{2} \vec{k}_{\|} \hat{\zeta}\left(\vec{k}_{\|}\right) \int d z^{\prime}\left[d_{y x} E_{x}^{(0)}+d_{y y} E_{y}^{(0)}+d_{y z} E_{z}^{(0)}\right] \delta\left(z^{\prime}\right)
\end{array}
$$

Next we have to calculate the components $E_{i}^{(0)}$ from the CPS theory for flat surfaces [3]. Since our interest is only to evaluate the expressions (17) and (18) on the $z$-axis with $(x, y)=(0,0)$, it is not difficult to verify from the original CPS theory that for an $x$-oriented dipole, only $\mathrm{E}_{\mathrm{x}}^{(0)}$ survives in (17) and for an y-oriented dipole, only $\mathrm{E}_{\mathrm{y}}^{(0)}$ contributes in (18) (See Appendix A). Hence, $\mathrm{E}_{i}^{\mathrm{rR}}$ in (17) and (18) evaluated at $(0,0, z)$ will take the following simple forms : 


$$
\begin{aligned}
& \mathrm{E}_{\mathrm{x}}^{\mathrm{rR}}(\mathrm{z}, \omega)=-\frac{\mathrm{k}^{2}}{16 \pi^{3}}[\varepsilon(\omega)-1] \int \mathrm{d}^{2} \overrightarrow{\mathrm{k}}_{\|} \hat{\zeta}\left(\overrightarrow{\mathrm{k}}_{\|}\right) \int \mathrm{d} \mathrm{z}^{\prime} \mathrm{d}_{\mathrm{xx}} \mathrm{E}_{\mathrm{x}}^{(0)}\left(\mathrm{z}^{\prime}\right) \delta\left(\mathrm{z}^{\prime}\right), \\
& \mathrm{E}_{\mathrm{y}}^{\mathrm{rR}}(\mathrm{z}, \omega)=-\frac{\mathrm{k}^{2}}{16 \pi^{3}}[\varepsilon(\omega)-1] \int \mathrm{d}^{2} \overrightarrow{\mathrm{k}}_{\|} \hat{\zeta}\left(\overrightarrow{\mathrm{k}}_{\|}\right) \int \mathrm{d} \mathrm{z}^{\prime} \mathrm{d}_{\mathrm{yy}} \mathrm{E}_{\mathrm{y}}^{(0)}\left(\mathrm{z}^{\prime}\right) \delta\left(\mathrm{z}^{\prime}\right),
\end{aligned}
$$

with $\mathrm{E}_{\mathrm{i}}^{(0)}=\mathrm{E}_{\mathrm{i}}^{\mathrm{in}}+\mathrm{E}_{\mathrm{i}}^{\mathrm{rF}}$ and can be expressed in terms of the "Sommerfeld integrals" as given in the CPS paper [3] :

$$
\begin{aligned}
& E_{i}^{\text {in }}\left(z^{\prime}, \omega\right)=\hat{e}_{i} \mu k^{3} \int_{0}^{\infty} d u \frac{u^{3}}{\ell_{1}} e^{k \ell_{1}\left(z^{\prime}-d\right)} \\
& E_{i}^{r F}\left(z^{\prime}, \omega\right)=\hat{e}_{i} \frac{\mu k^{3}}{2} \int_{0}^{\infty} d u \frac{u}{\ell_{1}}\left[\left(1-u^{2}\right) R^{\|}+R^{\perp}\right] e^{-k \ell_{1}\left(z^{\prime}+d\right)}
\end{aligned}
$$

Note that in the case of flat surface, there is no distinction between the $\mathrm{x}$-dipole case and the $\mathrm{y}$-dipole case. $\mathrm{R}^{\|}=\frac{\ell_{2}-\varepsilon \ell_{1}}{\ell_{2}+\varepsilon \ell_{1}} \quad$ and $\mathrm{R}^{\perp}=\frac{\ell_{1}-\ell_{2}}{\ell_{1}+\ell_{2}} \quad$ are the Fresnel coefficients with $\ell_{1}=-\mathrm{i} \sqrt{1-\mathrm{u}^{2}}$ and $\quad \ell_{2}=-\mathrm{i} \sqrt{\varepsilon-\mathrm{u}^{2}}$ Hence the integrals $\int d^{\prime}$ in Eqs. (19) and (20) can be evaluated to yield:

$$
\begin{aligned}
& \int d z^{\prime} d_{x x} E_{x}^{(0)}\left(z^{\prime}\right) \delta\left(z^{\prime}\right)=\frac{\mu k^{3}}{2} d_{x x}\left(\vec{k}_{\|}, \omega \mid z, 0\right) \int_{0}^{\infty} d u \frac{u}{\ell_{1}}\left[2 u^{2}+\left(1-u^{2}\right) R^{\|}+R^{\perp}\right] e^{-k \ell_{1} d} \\
& \int d z^{\prime} d_{y y} E_{y}^{(0)}\left(z^{\prime}\right) \delta\left(z^{\prime}\right)=\frac{\mu k^{3}}{2} d_{y y}\left(\vec{k}_{\|}, \omega \mid z, 0\right) \int_{0}^{\infty} d u \frac{u}{\ell_{1}}\left[2 u^{2}+\left(1-u^{2}\right) R^{\|}+R^{\perp}\right] e^{-k \ell_{1} d}
\end{aligned}
$$


Note that since both the dyadics and the components $E_{\mathbf{x}}^{(0)}$ and $E_{y}^{(0)}$ are continuous across the surface $z=0$, there is no ambiguity in carrying out the integral $\int d z^{\prime} \delta\left(z^{\prime}\right)$ to arrive at Eqs. (23) and (24). In comparison, for the case of a perpendicular dipole, such an integral must be handled with care since $\mathrm{E}_{\mathrm{z}}^{(0)}$ is not continuous at the boundary $[8,13]$. To proceed further with the calculation, we need the expressions for the Green dyadics from the MM paper [12] .

Following the appendix of the MM paper (see Appendix B), $d_{x x}$ and $d_{y y}$ can be represented as:

$$
\begin{aligned}
& d_{x x}\left(\vec{k}_{\|}, \omega \mid z, z^{\prime}=0\right)=\frac{4 \pi i}{k^{2} k_{\|}^{2}} e^{i k_{2} z}\left(\frac{k^{2} k_{y}^{2}}{k_{1}-k_{2}}-\frac{k_{1} k_{2} k_{x}^{2}}{k_{1}-\varepsilon k_{2}}\right), \\
& d_{y y}\left(\vec{k}_{\|}, \omega \mid z, z^{\prime}=0\right)=\frac{4 \pi i}{k^{2} k_{\|}^{2}} e^{i k_{2} z}\left(\frac{k^{2} k_{x}^{2}}{k_{1}-k_{2}}-\frac{k_{1} k_{2} k_{y}^{2}}{k_{1}-\varepsilon k_{2}}\right),
\end{aligned}
$$

where $k_{1}$ and $k_{2}$ are defined as :

$$
\begin{aligned}
& \mathrm{k}_{1}=-\left(\varepsilon \mathrm{k}^{2}-\mathrm{k}_{\|}^{2}\right)^{1 / 2} \\
& \mathrm{k}_{2}= \begin{cases}\left(\mathrm{k}^{2}-\mathrm{k}_{\|}^{2}\right)^{1 / 2}, & \mathrm{k}^{2}>\mathrm{k}_{\|}^{2} \\
\mathrm{i}\left(\mathrm{k}_{\|}^{2}-\mathrm{k}^{2}\right)^{1 / 2}, & \mathrm{k}^{2}<\mathrm{k}_{\|}^{2} .\end{cases}
\end{aligned}
$$

Furthermore, for a sinusoidal grating surface profile with

$$
\hat{\zeta}\left(\overrightarrow{\mathrm{k}}_{\|}\right)=(2 \pi)^{2} \zeta_{0} \delta\left(\overrightarrow{\mathrm{Q}}-\overrightarrow{\mathrm{k}}_{\|}\right) \quad, \quad \overrightarrow{\mathrm{Q}}=\mathrm{Q} \hat{\mathrm{e}}_{\mathrm{x}}
$$


the integrals $\int \mathrm{d}^{2} \overrightarrow{\mathrm{k}}_{\|}$in (17) and (18) can be evaluated (with the results in $(23)-(26)$ ) to give:

$$
\begin{aligned}
& \int d^{2} \vec{k}_{\|} \hat{\zeta}\left(\vec{k}_{\|}\right) d_{x x}\left(\vec{k}_{\|}, \omega \mid z\right)=-i \frac{16 \pi^{3} \zeta_{0}}{k^{2}} \frac{k_{1} k_{2}}{k_{1}-\varepsilon k_{2}} e^{i k_{2} z} \\
& \int d^{2} \vec{k}_{\|} \hat{\zeta}\left(\vec{k}_{\|}\right) d_{y y}\left(\vec{k}_{\|}, \omega \mid z\right)=i \frac{16 \pi^{3} \zeta_{0}}{k_{1}-k_{2}} e^{i k_{2} z}
\end{aligned}
$$

Substituting (23), (24), (29), and (30) into (19) and (20), we finally obtain the first order roughness contribution to the reflected field at the dipole site $(z=d)$ in the following form :

$\mathrm{E}_{\mathrm{x}}^{\mathrm{rR}}=\frac{\mathrm{i} \mu \mathrm{k}^{3}}{2}(\varepsilon-1) \zeta_{0} \frac{\mathrm{k}_{1} \mathrm{k}_{2}}{\mathrm{k}_{1}-\varepsilon \mathrm{k}_{2}} \mathrm{e}^{\mathrm{i} \mathrm{k}_{2} \mathrm{~d}} \int_{0}^{\infty} \mathrm{du} \frac{\mathrm{u}}{\ell_{1}}\left[2 \mathrm{u}^{2}+\left(1-\mathrm{u}^{2}\right) \mathrm{R}^{\|}+\mathrm{R}^{\perp}\right] \mathrm{e}^{-\mathrm{k} \ell_{1} \mathrm{~d}}$

$$
\mathrm{E}_{\mathrm{y}}^{\mathrm{rR}}=-\frac{\mathrm{i} \mu \mathrm{k}^{5}}{2}(\varepsilon-1) \zeta_{0} \frac{1}{\mathrm{k}_{1}-\mathrm{k}_{2}} \mathrm{e}^{\mathrm{i} \mathrm{k}_{2} \mathrm{~d}} \int_{0}^{\infty} \mathrm{du} \frac{\mathrm{u}}{\ell_{1}}\left[2 \mathrm{u}^{2}+\left(1-\mathrm{u}^{2}\right) \mathrm{R}^{\|}+\mathrm{R}^{\perp}\right] \mathrm{e}^{-\mathrm{k} \ell_{1} \mathrm{~d}}
$$


IV. MOLECULAR FLUORESCENCE CHARACTERISTICS

Combining the results from the above section and Eqs. (1) and (2), the modified emission frequency and the decay rate can be obtained in the following form :

$$
\begin{aligned}
& \frac{\Delta \omega}{\gamma_{0}}=-\frac{3 \mathrm{q}}{4 \mathrm{k}^{3}} \operatorname{ReG}_{\mathrm{i}} \\
& \frac{\gamma}{\gamma_{0}}=1+\frac{3 \mathrm{q}}{2 \mathrm{k}^{3}} \operatorname{ImG}_{\mathrm{i}}
\end{aligned}
$$

where $G_{i}=\frac{E_{i}^{r}}{\mu}$ is the reflected field acting on the dipole per unit dipole moment of the admolecule with the subscript $i$ indicating the orientation of the dipole with respect to the surface. To be complete and selfcontained, the full set of solutions for $G_{i}$ to first order roughness for a grating surface obtained in both the dynamic and the approximated static image theories are given below. Thus let us write

$$
\mathrm{G}_{\mathrm{i}}=\mathrm{G}_{\mathrm{i}}^{\mathrm{F}}+\mathrm{G}_{\mathrm{i}}^{\mathrm{R}}
$$

where $G_{i}^{F}$ is given by the CPS theory in the dynamical approach as follows :

For a parallel dipole (for both $x$ and $y$ ) :

$$
G_{x, y}^{F}=\frac{k^{3}}{2} \int_{0}^{\infty} d u \frac{u}{\ell_{1}}\left[\left(1-u^{2}\right) R^{\|}+R^{\perp}\right] e^{-2 k \ell_{1} d},
$$

For a perpendicular dipole $(z)$ : 


$$
G_{z}^{F}=-k^{3} \int_{0}^{\infty} d u \frac{u^{3}}{\ell_{1}} R e^{-2 k \ell_{1} d}
$$

$\mathrm{G}_{\mathrm{i}}^{\mathrm{R}}$ is then obtained from the results in Eqs. (31) and together with previous results [8] as follows:

$$
\begin{aligned}
\mathrm{G}_{\mathrm{x}}^{\mathrm{R}} & =\frac{\mathrm{ik}{ }^{3}}{2}(\varepsilon-1) \zeta_{0} \frac{\mathrm{k}_{1} \mathrm{k}_{2}}{\mathrm{k}_{1}-\varepsilon \mathrm{k}_{2}} \mathrm{e}^{\mathrm{i} \mathrm{k}_{2} \mathrm{~d}} \int_{0}^{\infty} \mathrm{du} \frac{\mathrm{u}}{\ell_{1}}\left[2 \mathrm{u}^{2}+\left(1-\mathrm{u}^{2}\right) \mathrm{R}^{\|}+\mathrm{R}^{\perp}\right] \mathrm{e}^{-\mathrm{k} \ell_{1} \mathrm{~d}}, \\
\mathrm{G}_{\mathrm{y}}^{\mathrm{R}} & =-\frac{\mathrm{ik}}{2}(\varepsilon-1) \zeta_{0} \frac{1}{\mathrm{k}_{1}-\mathrm{k}_{2}} \mathrm{e}^{\mathrm{i} \mathrm{k}_{2} \mathrm{~d}} \int_{0}^{\infty} \mathrm{du} \frac{\mathrm{u}}{\ell_{1}}\left[2 \mathrm{u}^{2}+\left(1-\mathrm{u}^{2}\right) \mathrm{R}^{\sharp}+\mathrm{R}^{\perp}\right] \mathrm{e}^{-\mathrm{k} \ell_{1} \mathrm{~d}}, \\
\mathrm{G}_{\mathrm{z}}^{\mathrm{R}} & =-i \mathrm{k}^{3}(\varepsilon-1) \zeta_{0} \frac{\mathrm{Q}^{2}}{\mathrm{k}_{1}-\varepsilon \mathrm{k}_{2}} \mathrm{e}^{\mathrm{i} \mathrm{k}_{2} \mathrm{~d}} \int_{0}^{\infty} \mathrm{du} \frac{\mathrm{u}^{3}}{\ell_{1}}\left(1-\mathrm{R}^{\mathrm{i}}\right) \mathrm{e}^{-\mathrm{k} \ell_{1} \mathrm{~d}},
\end{aligned}
$$

where $k_{1}$ and $k_{2}$ in $(38)-(40)$ are defined as :

$$
\begin{aligned}
& \mathrm{k}_{1}=-\left(\varepsilon \mathrm{k}^{2}-\mathrm{Q}^{2}\right)^{1 / 2} \\
& \mathrm{k}_{2}= \begin{cases}\left(\mathrm{k}^{2}-\mathrm{Q}^{2}\right)^{1 / 2}, & \mathrm{k}^{2}>\mathrm{Q}^{2} \\
\mathrm{i}\left(\mathrm{Q}^{2}-\mathrm{k}^{2}\right)^{1 / 2}, & \mathrm{k}^{2}<\mathrm{Q}^{2} .\end{cases}
\end{aligned}
$$

In the long wavelength limit, one can also apply the static image theory to obtain the following results [7] : $\mathrm{G}_{\mathrm{x}}=\mathrm{G}_{\mathrm{x}}^{\mathrm{F}}+\mathrm{G}_{\mathrm{x}}^{\mathrm{R}}=$ $=\frac{(\varepsilon-1)}{(\varepsilon+1)}\left(\frac{1}{8 d^{3}}\right)+\frac{4 \zeta}{\pi} \frac{\varepsilon-1}{(\varepsilon+1)^{2}} \int_{0}^{\infty} d u \int_{0}^{\infty} d v \frac{u^{2}-\frac{Q^{2}}{4}}{f g}(\varepsilon f g+h) \exp [-(f+g) d]$

$\mathrm{G}_{\mathrm{y}}=\mathrm{G}_{\mathrm{y}}^{\mathrm{F}}+\mathrm{G}_{\mathrm{y}}^{\mathrm{R}}=$ 
$=\frac{(\varepsilon-1)}{(\varepsilon+1)}\left(\frac{1}{8 d^{3}}\right)+\frac{4 \zeta}{\pi} \frac{\varepsilon-1}{(\varepsilon+1)^{2}} \int_{0}^{\infty} d u \int_{0}^{\infty} d v \frac{v^{2}}{f g}(\varepsilon f g+h) \exp [-(f+g) d]$

$\mathrm{G}_{\mathrm{z}}=\mathrm{G}_{\mathrm{z}}^{\mathrm{F}}+\mathrm{G}_{\mathrm{z}}^{\mathrm{R}}=$

$=\frac{\varepsilon-1}{\varepsilon+1}\left(\frac{1}{4 \mathrm{~d}^{2}}\right)+\frac{4 \zeta}{\pi} \frac{\varepsilon-1}{(\varepsilon+1)^{2}} \int_{0}^{\infty} \mathrm{du} \int_{0}^{\infty} \mathrm{dv}(\varepsilon f g+h) \exp [-(\mathrm{f}+\mathrm{g}) \mathrm{d}]$

where $f, g, h$ are functions of $u, v$, given by

$$
\begin{aligned}
& f(u, v)=\left(\left(u+\frac{Q}{2}\right)^{2}+v^{2}\right)^{1 / 2} \\
& g(u, v)=\left(\left(u-\frac{Q}{2}\right)^{2}+v^{2}\right)^{1 / 2} \\
& h(u, v)=u^{2}+v^{2}-\frac{Q^{2}}{4}
\end{aligned}
$$

Another limiting case of interest is the perfect reflecting (conducting) limit in which both the Fresnel coefficients $R^{\|}$and $R^{\perp}$ are set to -1 [3]. For flat substrate surfaces, this case was considered in the original works by CPS and others for both the parallel and perpendicular dipoles. It is therefore tempting to study the same limiting case for rough surfaces following the above formulation. However, on a careful examination, it can be seen that this is illegitimate in the present approach since the original MM perturbation theory [12] to 
lowest order is valid only for the case when the roughness amplitude $\zeta$ is much less than the skin depth of the substrate metal. This can be seen from the original expansion of the dielectric function $\varepsilon(z, \omega)$ in terms of $\zeta$ (Eq.4) in which the expansion will lose meaning for a perfect conducting substrate with $\varepsilon \rightarrow-\infty$. Thus for a perfect conductor with a zero skin depth, the perfect reflecting limit cannot be taken in our present perturbation theory for rough surfaces as was mistakenly done in a previous work [14]. So it seems that for the perfect reflecting limit, which is a relatively simple case with a flat substrate surface, the molecular fluorescence properties must be studied non-perturbatively in the case with a rough surface [15]. 


\section{METHODOLOGY}

The numerical analysis of the equations for the frequency-shift and the modified decay rate was carried out on an IBM PC using the FORTRAN programming language. The primary program used for these calculations is given in Appendix $C$. It should be noted that this program was modified many times in order to obtain the desired data. However, these modifications were primarily parameter based and to avoid redundancy, this will be the only program included with this thesis.

The integrals invloved in Eqs.(36)-(40) contain a singularity, at 1 , and must be handled with care in the numerical analysis. To do this, a FORTRAN scientific package(IMSL) was used which allows the calculation of an integral with a singularity. The function called in the program is the DQDAGP function.

To assure a valid approach using this program, many of the results previously obtained were verified. Also, unless otherwise stated, the functional parameters for the following numerical analysis remained constant. The frequency of emission was $2.5 \mathrm{eV}$, the dipole-substrate distance was $10 \mathrm{~nm}$, and the roughness parameters always kept at values that satisfied the form $Q \zeta_{0}=0.02$. 


\section{NUMERICAL RESULTS AND DISCUSSION}

The above theory of interaction between an emitting dipole and a periodic conducting surface has been applied to compute the fluorescence characteristics of molecules at a grating surface : the $\mathrm{x}$ orientation being that in which the dipole is oriented along the direction of the grating, the $y$ orientation with the dipole situated parallel to the grooves, and the $z$ orientation with the dipole perpendicular to the substrate. Figure 2 shows the geometry of the system. Listed are the dipole-substrate distance $d$, the roughness parameters $\zeta_{0}$ (amplitude) and $Q$ (grating wave vector), and the dielectric constant of the substrate, $\varepsilon(\omega)$, with the dipole located in vacuum. All the computations were carried out in the limit of shallow roughness with $Q \zeta_{0}=0.02$ and the substrate taken as silver whose optical properties can be obtained from the literature [16]. Figure 3 shows the comparison of the static and dynamic theories by plotting the imaginary part of the roughness contribution, $\mathrm{ImG}^{\mathrm{R}}$, against the distance d for the three dipole orientations. It can be seen that for the $y$ and $z$ orientations, the two theories compare well at close distances, while at greater distances, the static (image) theory is consistently below that of the dynamical theory. This observation is in agreement with previous work for flat surfaces [1.7] and vertical dipoles 

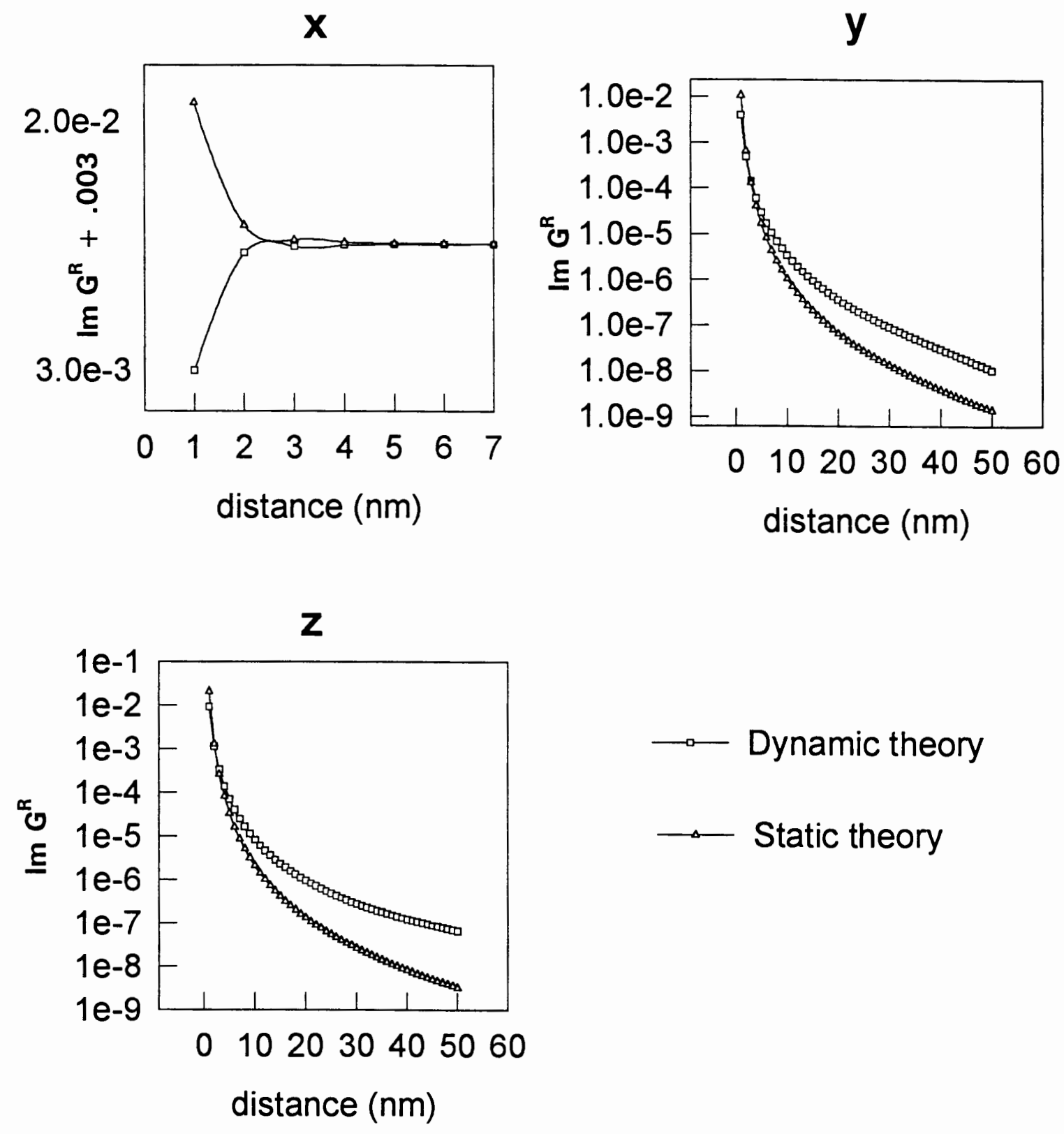

Fig.3 Comparison between the static and dynamic theories by plotting Im $\mathrm{G}^{\mathrm{R}}$ in Eqs. (38-40) and in Eqs. (42-44) as a function of the dipole-substrate distance.

The labels $x, y$, and $z$ indicate the three orientations of the molecule. 
at grating surfaces [8]. However, the $\mathrm{x}$ orientation differs from the other two in that the dynamic theory gives negative values for $\mathrm{ImG}^{\mathrm{R}}$ at close distances. The displayed graphic was offset by a constant so it could be shown on a logarithmic plot. The dramatic difference in the $\mathrm{x}$ orientation possibly has its origin from the radiative transfer between the molecule and the surface which can only be accounted for in a dynamic theory [11]. It is well known that the distance dependence of the decay rates for the case of flat surfaces is highly sensitive to the molecular orientation, due mainly to the predominance of radiative transfer at relatively far distances for the perpendicular dipoles but much less for the parallel dipoles [3]. In the presence of roughness, this issue is further complicated by the fact that non-radiative transfer can be transformed back to a radiative one due to the re-coupling of evanescent surface modes to radiative modes. This re-coupling mechanism is particularly significant for the $\mathrm{x}$ dipole being oriented along the direction of the grating wave vector.

Figure 4 shows the results obtained for the decay rate at a rough surface, normalized to the flat surface values, as a function of distance. The curves have varying emitting frequencies and they all tend to unity at far distances as expected. At closer distances ( $d<25 \mathrm{~nm}$ ), 
$\mathbf{x}$
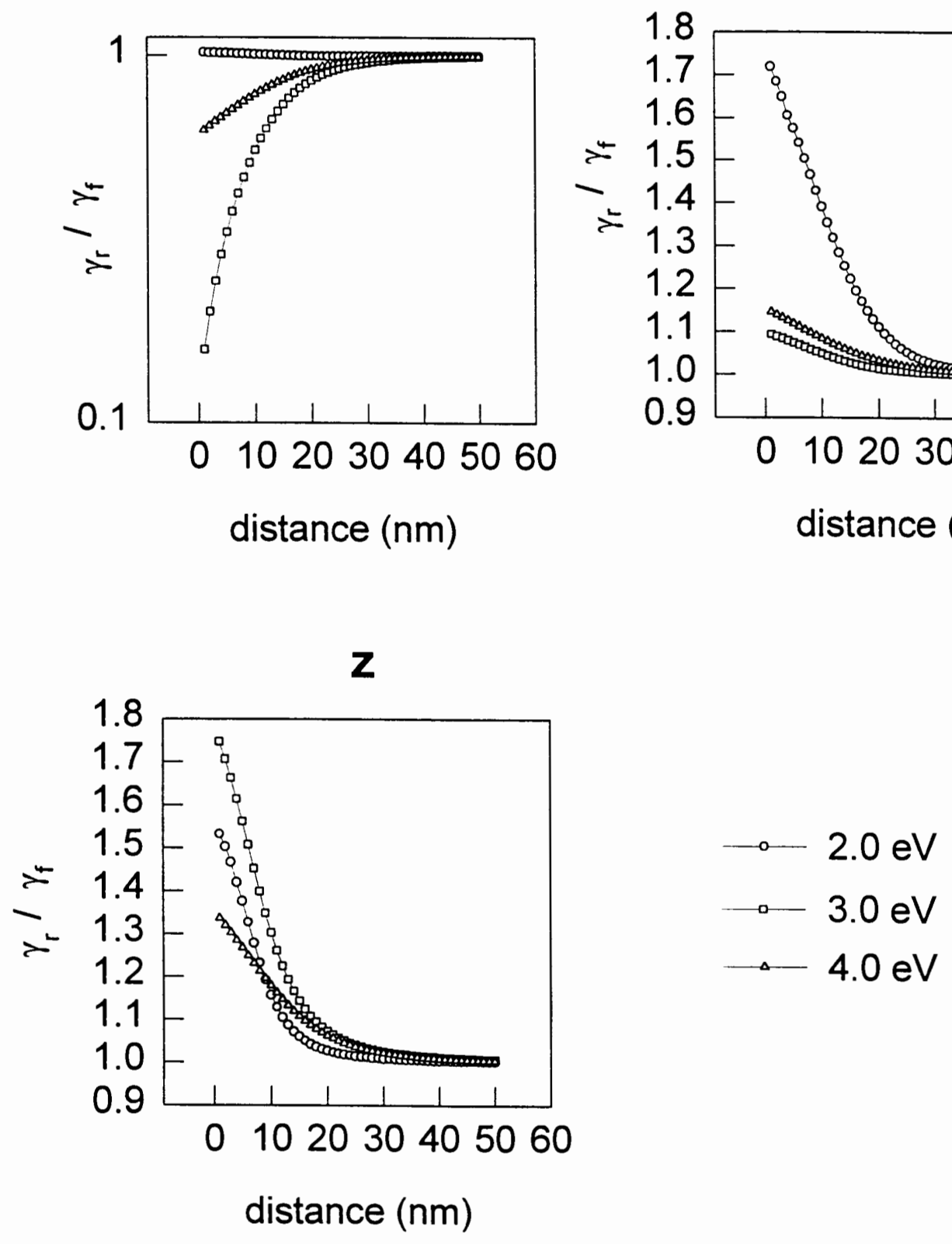

Fig.4. Ratio of the decay rate at a rough surface to that at a flat surface as a function of distance, plotted for three different emission frequencies.

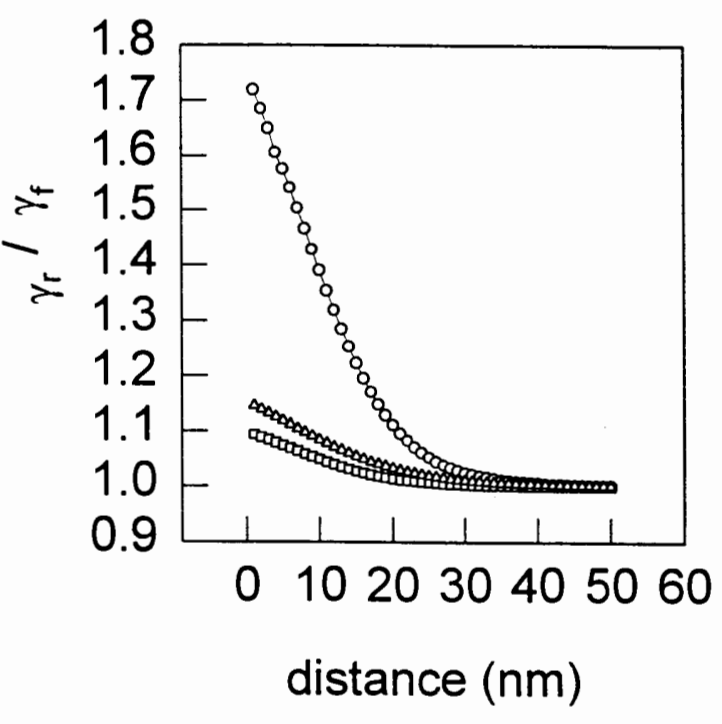


the results are sensitive to both the dipole orientation and the emission frequency. The most interesting observation from these is that the presence of roughness can both enhance or suppress (!) the flat surface decay rates for admolecules, in agreement with previous remarks $[7,8,11]$ and is somewhat unexpected from other investigations [6]. Specifically, we note that within this frequency range, the grating roughness tends to decrease the flat surface decay values for the $\mathrm{x}$-oriented dipoles while enhancing those for the $y^{-}$and $z$-oriented dipoles. In general, both enhancement and diminution can occur for all the $\mathrm{x}^{-}, \mathrm{y}^{-}$, and $\mathrm{z}^{-}$dipoles at different emission frequencies. In fact, for all dipole orientations these effects were seen in this study. However, with the many parameters which can be varied in the calculations, it was convenient to maintain a consistency with the majority of the plotted data and thus, the presentation of this particular case.

Having studied the effect of the roughness with respect to a flat surface, the rest of the calculations will normalize the emission characteristics in the presence of the grating with respect to those for a free molecule. Figure 5 shows the total ("flat + rough ") decay rate normalized to that of a free molecule. Notice that while the perpendicular ( $z$ ) dipole always has its surface-induced decay rate greater than that of a free 

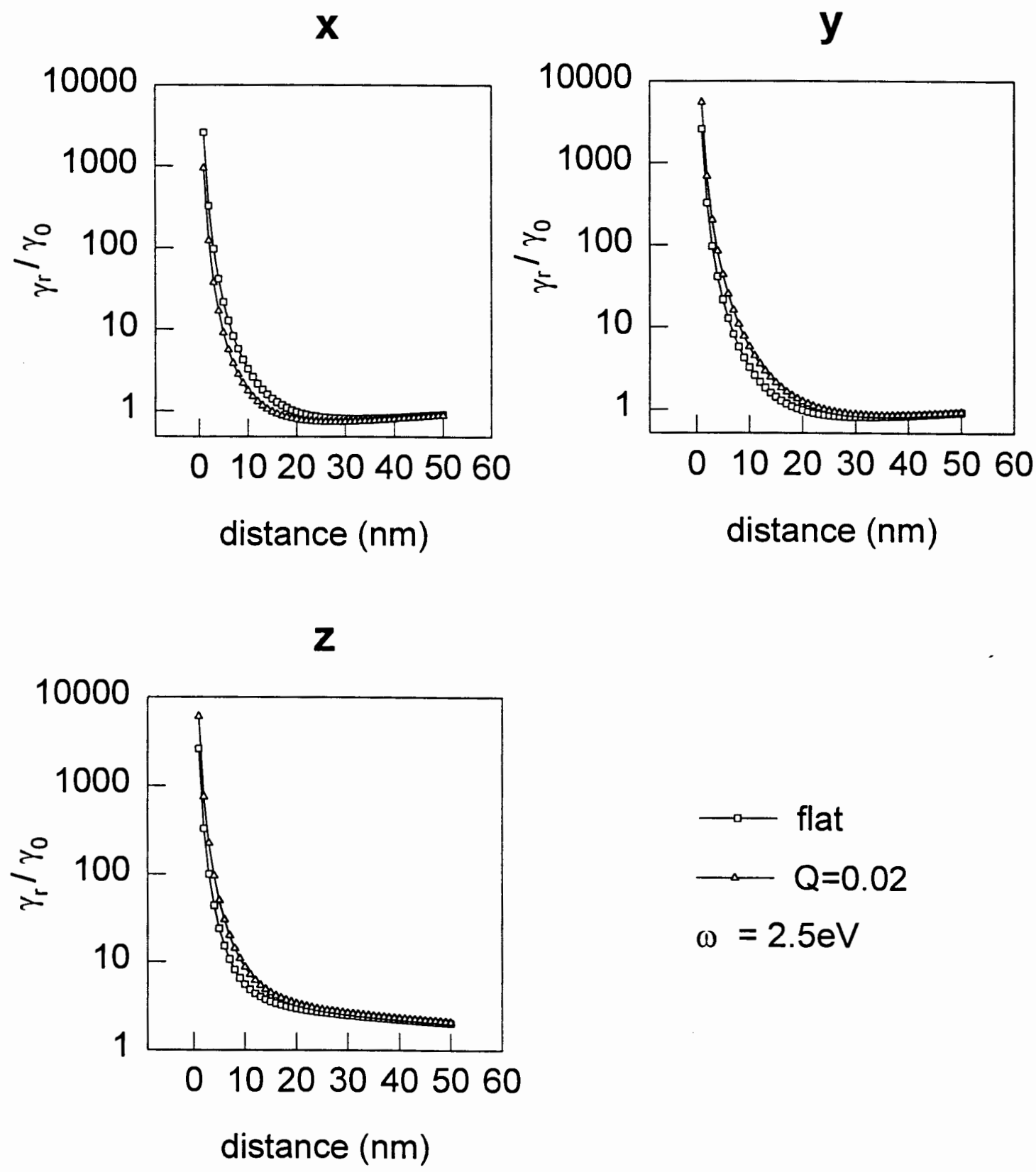

Fig.5 Similar to Fig.4, except the surface-induced decay rate is normalized to the free molecule value, rather than to the flat surface value. The case for a flat surface and that for a grating with $\mathrm{Q}=0.02 \mathrm{~nm}^{-1}$ are shown for the emission frequency at $2.5 \mathrm{eV}$. 
molecule, the parallel ( $x$ and $y$ ) dipoles can have the induced rates less than the free molecule rate at relatively far distances. This is well known with flat surfaces and is due again to the aforementioned relatively small radiative transfer for parallel dipoles at far distances as a result of the "destructive interference effect" between the radiating dipole and its image [3]. However, in the presence of roughness, the $x$-dipole has its decay rate less than the flat surface values since the roughness contribution is negative (see Fig. 3) while the y-dipole behaves just the opposite. This leads to the result that the total decay rate for the $\mathrm{x}$-dipole at a grating surface can become smaller than the free molecule rate at closer distances (reduced from about $20 \mathrm{~nm}$ to 10 nm) from the surface! The result will be somewhat dramatic if the drop below the free molecule rate can occur at even closer distances (say, $d<5 \mathrm{~nm}$ ) within which the presence of the surface is traditionally thought to certainly increase the damping of the molecule due to non-radiative transfer. Although it cannot be demonstrated from the present perturbative calculation, this result surely leaves open the possibility that the presence of roughness can be exploited to lengthen the lifetimes of the admolecules (even beyond its free molecule value!) by manipulating the orientations of the molecules. This possibility could have significant 
implications to performing photochemistry at rough metallic surfaces [11].

Figure 6 shows the same total decay rate as a function of emission frequencies. It should be pointed out that there exists two "resonance structures" in this perturbative formalism as can be seen from Eqs. (38)-(40). One structure depends on the presence of the roughness (the morphology- or Q-dependent resonance) as can be seen from the terms $\sim 1 /\left(k_{1}-\varepsilon k_{2}\right)$ or $\sim 1 /\left(k_{1}-k_{2}\right)$. The other is just like the flat surface case through the factors $\mathrm{R}^{\perp}$ and $\mathrm{R}^{\|}$which imply a surface plasmon resonance at about $3.7 \mathrm{eV}$ for a silver substrate. These two structures interplay with each other in a complicated manner depending on the value of $Q$, the distance, as well as the molecular orientation. As a result, a kind of "shoulder peak" is manifested in some of these plots [9]. The negative results shown in the $\mathrm{x}$ orientation case are obviously unacceptable but are most likely due to the limitation of this perturbative approach.

The remaining two graphs show the result for frequency-shift calculations for different molecular orientations. Aside from the general surface-induced redshifts appreciable at close distances and low emission frequencies as observed before [10], the following interesting features should be noted. Figure 7 shows the 

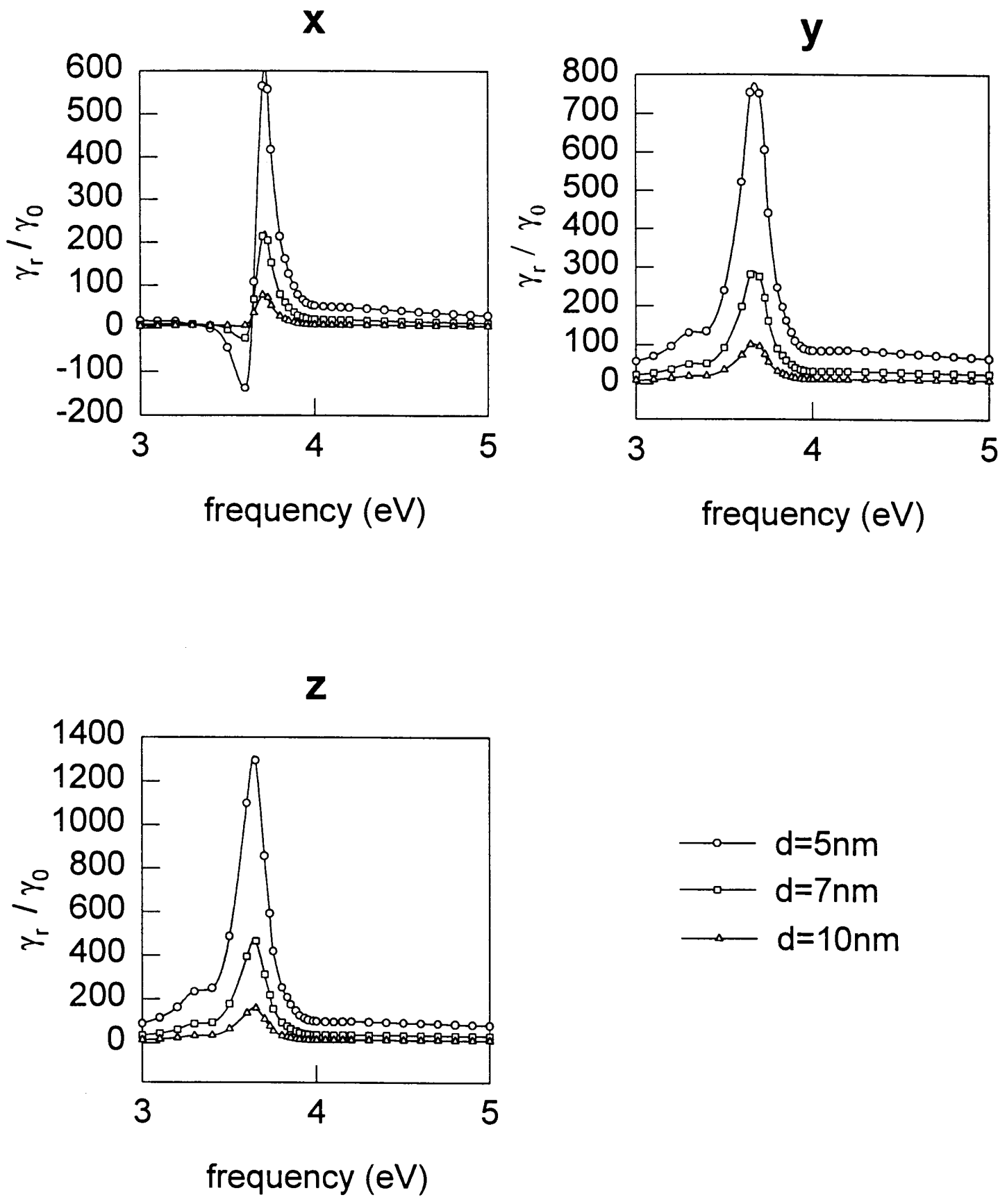

Fig. 6. Normalized decay rate spectrum for a grating surface with $Q=0.02 \mathrm{~nm}^{-1}$ as a function of emission frequency at three different molecule-surface distances. 

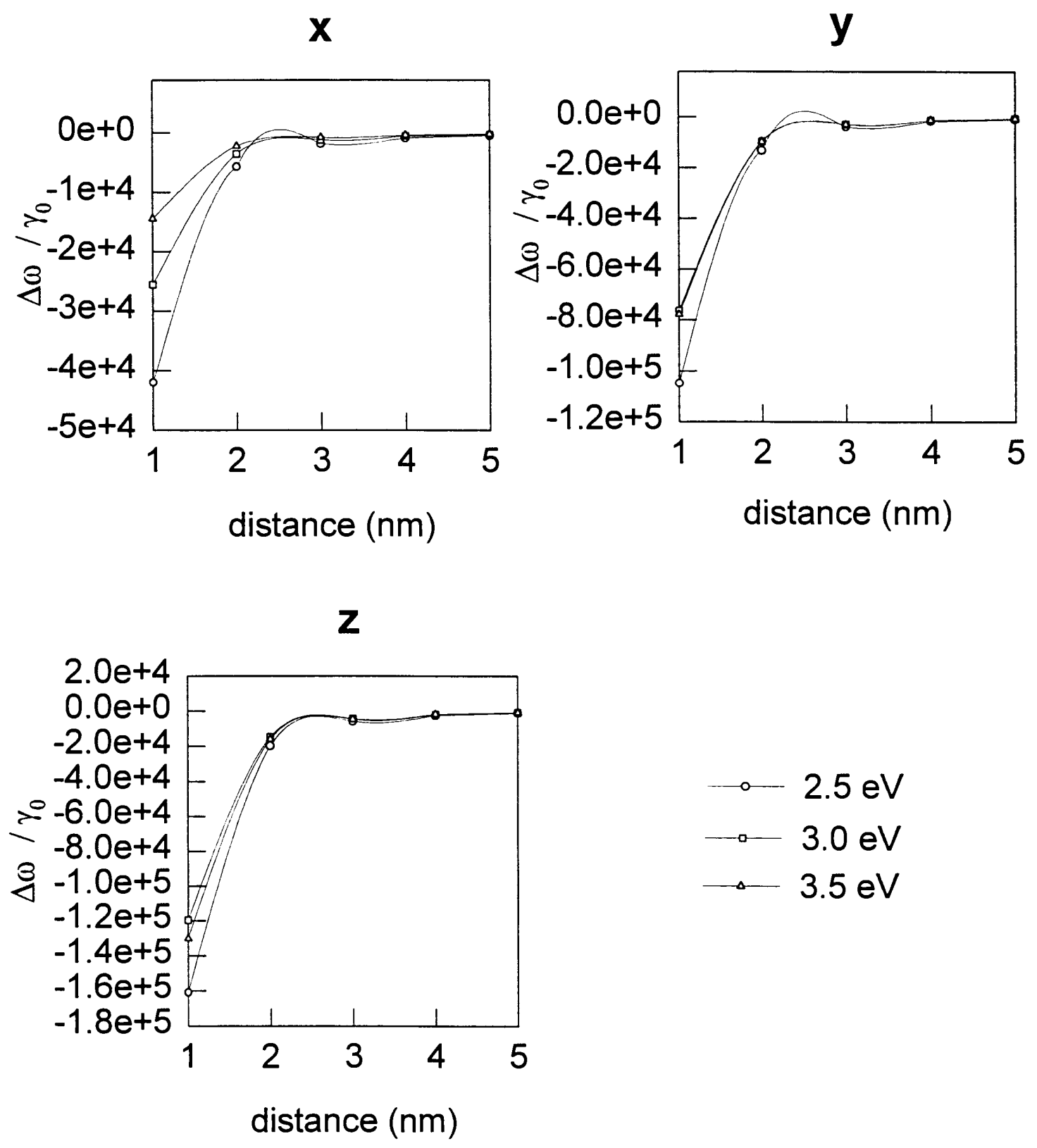

Fig. 7. Normalized molecular frequency-shifts for a grating surface with $Q=0.02 \mathrm{~nm}^{-1}$ as a function of distance for three different emission frequencies. 
shifts as a function of distance for three different emitting Erequencies. Notice that while the $\mathrm{x}$-dipole has in general smaller red-shifts in its frequency, it is also more sensitive to the change of emitting frequency as compared to the cases with the $y$ and $z$ dipoles. Furthermore, it is interesting to note that the surface effects on the emission frequency drop down much more rapidly than as in the case for the decay rates as the molecule is located farther away from the surface. Figure 8 shows the result as a function of the emission frequency at a fixed distance $d=10 \mathrm{~nm}$. Again, we see the extra Qdependent resonance structure showing up for values of $k$ close to $Q\left(Q=0.02 \mathrm{~nm}^{-1}\right)$ in the case of the $x$ - and $z$ dipoles. The disappearance of the effect at high emission Erequencies probably has to do with the "overall cancellation" of the contributions due to the misaligned image dipoles which become more significant when the source dipole oscillates very rapidly. 

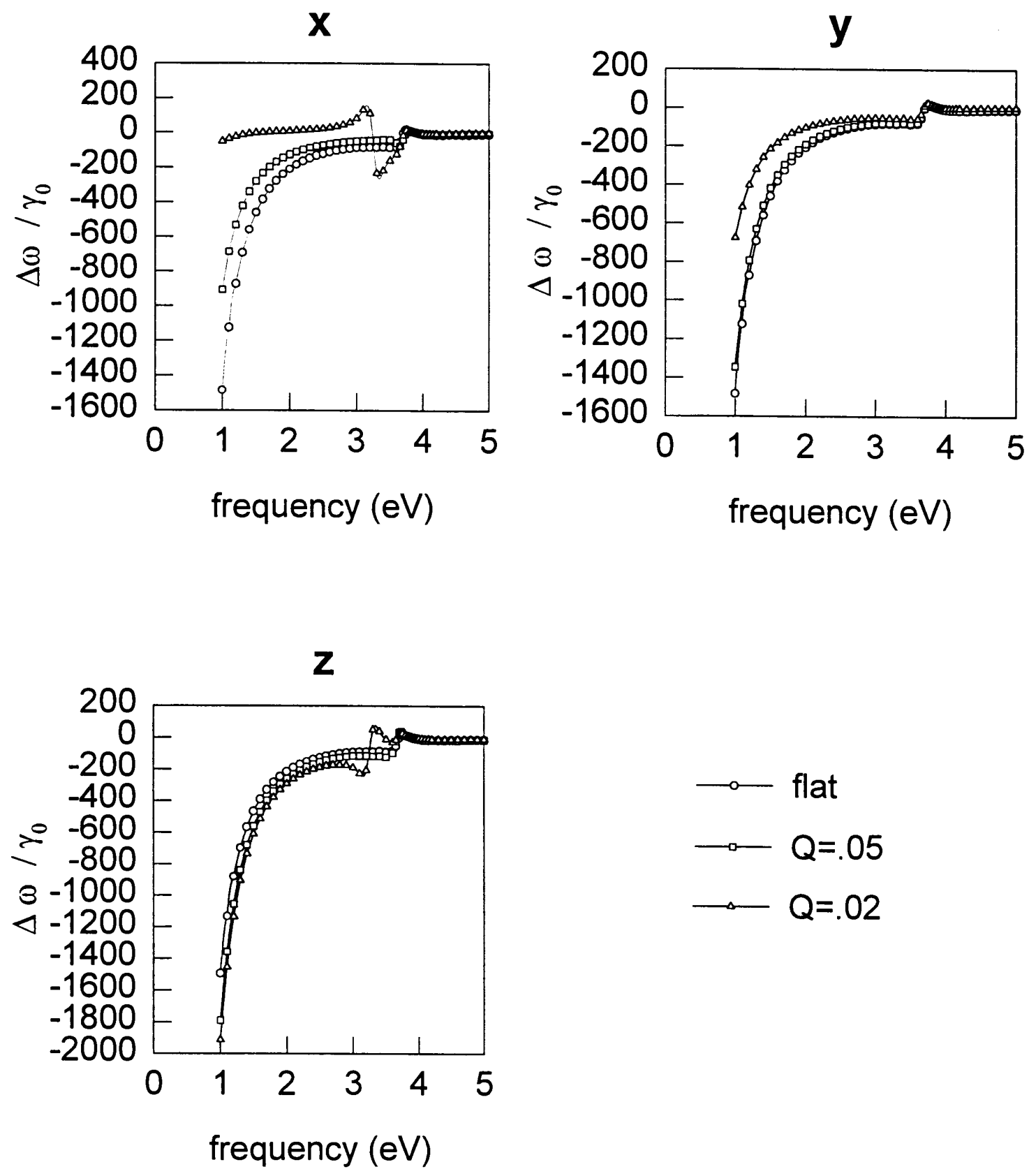

Fig. 8. Same as in Fig. 7, except for a plot as a function of emission frequency at a fixed distance of $10 \mathrm{~nm}$.

The results are shown for a flat surface, a grating surface with $Q=0.02 \mathrm{~nm}^{-1}$, and one with $\mathrm{Q}=0.05 \mathrm{~nm}^{-1}$, respectively. 


\section{CONCLUSION}

The dynamical theory for the effects on dipole emission near a rough conducting substrate has been expanded to include cases where the dipole has parallel and perpendicular orientations. The possible effects as functions of dipole distance from the substrate, dipole emission frequency, dipole orientation, and surface roughness have been explored. It has been shown that small changes in these parameters can affect the decay rate and frequency-shift in most cases. Although this present theory is limited by its perturbative approach and shallow-roughness approximation, the importance of establishing a dynamic theory for this phenomenon has been illustrated, with the hope that future more accurate (e.g. non-perturbative) approach will become available. Among other results as already elaborated in the above section, it should be noted that the present study implies an important modification in the usual step taken to compare experimental and theoretical results by "averaging" the theoretical calculated values over the orthogonal molecular orientations. For example, for decay rate ( $\gamma$ ) calculations, it is a common practice to compare $\gamma \equiv \gamma_{\perp} / 3+2 \gamma_{\|} / 3$ with experimental measurements. However, for patterned surfaces, the above results show that it may be more reasonable to compare the measurements with the 
calculated $\gamma \equiv\left(\gamma_{\mathrm{x}}+\gamma_{\mathrm{y}}+\gamma_{\mathrm{z}}\right) / 3$. Furthermore, it will also be interesting if future experimental evidence can indeed demonstrate the possibility of exploiting patterned surface roughness to lengthen the molecular lifetimes relative to those of a free molecule as well as those with a flat substrate surface. 


\section{REFERENCES}

[1] H. Kuhn, J. Chem. Phys. 53, 101 (1970).

[2] K. H. Drexhage, in Progress in Optics XII, edited by E. Wolf (North-Holland, Amsterdam, 1974), p.165 ff.

[3] R. R. Chance, A. Prock, and R. Silbey, Adv. Chem. Phys. 37, 1 (1978).

[4] A. Sommerfeld, Ann. Phys. (Leipzig) 28, 665 (1909); 81, 1135 (1926).

[5] R. Rossetti and L. E. Brus, J. Chem. Phys. 73, 572 (1980); 76, 1146 (1982);

A. P. Alivisatos, D. H. Waldeck, and C. B. Harris, J. Chem. Phys. 82, 541 (1985).

[6] J. Arias, P. K. Aravind, and H. Metiu, Chem. Phys. Lett. 85, 404 (1982).

[7] P. T. Leung, Z. C. Wu, D. A. Jelski, and T. F. George, Phys. Rev. B 36, 1475 (1987).

[8] P. T. Leung and T. F. George, Phys. Rev. B 36, 4664 (1987).

[9] P. T. Leung, Y. S. Kim, and T. F. George, Phys. Rev. B 38, 10032 (1988).

[10] M.H. Hider and P. T. Leung, Phys. Rev. B 44, 3262 (1991).

[11] For recent review, see, e.g., P. T. Leung and T. F. George, J. Chim. Phys. 92, 226 (1995).

[12] A. Maradudin and D. L. Mills, Phys. Rev. B 11, 1392 (1975).

[13] G. S. Agarwal, Phys. Rev. B 14, 846 (1976).

[14] The results for a perfect conducting substrate presented in Ref. [10] are wrong [ i.e. Eq. (6) and Figs. (4) and (5) ]. The factor $(\varepsilon-1) /\left(\mathrm{k}_{1}-\varepsilon \mathrm{k}_{2}\right)$ appeared in Eq. (6) should also have been considered in the perfect conducting limit. 
[15] See, e.g., the recent article by A. A. Maradudin, A. V. Shchegrov, T. A. Leskova, Opt. Commun. 135, 352 (1997) for the treatment of light scattering, and references therein.

[16] E. D. Palik ed., Handbook of Optical Constants of Solids vol. 1 (Academic, New York, 1985).

[17] P. T. Leung, T. F. George, and Y. C. Lee, J. Chem. Phys. 86, 7227 (1987). 


\section{APPENDIX A}

Following Sommerfeld, the Hertz vector for a parallel dipole (along $\mathbf{x}$ ) above the substrate is as follows:

$$
\begin{aligned}
\vec{\Pi}_{1}= & \hat{e}_{x} \mu k_{1} \int_{0}^{\infty}\left(e^{ \pm \ell_{1}(z-d)}+f_{1} e^{-\ell_{1} z}\right) \frac{u}{\ell_{1}} J_{0}(u r) d u+\hat{e}_{z} \mu k_{1} \frac{x}{r} \int_{0}^{\infty} g_{1} e^{-\ell_{1} z} J_{1}(u r) d u \\
& \text { (incident }+ \text { reflected) } \Pi_{x}+\text { (reflected) } \Pi_{z}
\end{aligned}
$$

where

$$
\mathrm{f}_{1}=\mathrm{R}^{\perp} \mathrm{e}^{-\ell_{1} \hat{\mathrm{d}}} \quad \text { and } \quad \mathrm{g}_{1}=\left(\mathrm{R}^{\|}-\mathrm{R}^{\perp}\right) \mathrm{e}^{-\ell_{1} \hat{\mathrm{d}}},
$$

with

$$
\mathrm{R}^{\sharp}=\frac{\ell_{2}-\varepsilon \ell_{1}}{\ell_{2}+\varepsilon \ell_{1}} \quad \text { and } \quad \mathrm{R}^{\perp}=\frac{\ell_{1}-\ell_{2}}{\ell_{1}+\ell_{2}}
$$

From this, the electric fields can be obtained by:

$$
\overrightarrow{\mathrm{E}}=\mathrm{k}^{2} \vec{\Pi}+\vec{\nabla} \vec{\nabla} \cdot \vec{\Pi}
$$

For $\vec{\Pi}=\left(\Pi_{x}, \Pi_{z}\right)$ and $k=k_{1}$ we get the following field components :

$$
\begin{aligned}
& \mathrm{E}_{\mathrm{x}}=\mathrm{k}_{1}^{2} \Pi_{\mathrm{x}}+\frac{\partial}{\partial \mathrm{x}}(\vec{\nabla} \cdot \vec{\Pi})=\mathrm{k}_{1}^{2} \Pi_{\mathrm{x}}+\left(\frac{\partial^{2} \Pi_{\mathrm{x}}}{\partial \mathrm{x}^{2}}+\frac{\partial^{2} \Pi_{\mathrm{z}}}{\partial \mathrm{x} \partial \mathrm{z}}\right) \\
& \mathrm{E}_{\mathrm{y}}=\frac{\partial}{\partial \mathrm{y}}(\vec{\nabla} \cdot \vec{\Pi})=\frac{\partial^{2} \Pi_{\mathrm{x}}}{\partial \mathrm{y} \partial \mathrm{x}}+\frac{\partial^{2} \Pi_{\mathrm{z}}}{\partial \mathrm{y} \partial \mathrm{z}} \\
& \mathrm{E}_{\mathrm{z}}=\mathrm{k}_{1}^{2} \Pi_{\mathrm{z}}+\frac{\partial}{\partial \mathrm{z}}(\vec{\nabla} \cdot \vec{\Pi})=\mathrm{k}_{1}^{2} \Pi_{\mathrm{z}}+\left(\frac{\partial^{2} \Pi_{\mathrm{x}}}{\partial \mathrm{x} \partial \mathrm{z}}+\frac{\partial^{2} \Pi_{\mathrm{z}}}{\partial \mathrm{z}^{2}}\right)
\end{aligned}
$$

Thus, using the components $\Pi_{x}$ and $\Pi_{z}$ one can find expressions for $E_{x}^{(0)}, E_{y}^{(0)}$, and $E_{z}^{(0)}$. However, my interest is limited to the fields at $(0,0, z)$, i.e. on the $z$-axis with 
$\overrightarrow{\mathrm{r}}_{\|} \rightarrow 0$. By symmetry it is easy to see that $E_{y}$ and $E_{z}$ vanish for a dipole oriented in the $\mathrm{x}$ direction. This will be explicitly demonstrated below.

First we recall some useful rules for Bessel functions.

$$
\begin{aligned}
& \text { (a) } \mathrm{J}_{\mathrm{p}}^{\prime}(\mathrm{x})=-\frac{\mathrm{p}}{\mathrm{x}} \mathrm{J}_{\mathrm{p}}(\mathrm{x})+\mathrm{J}_{\mathrm{p}-1}(\mathrm{x}) \\
& \text { (b) } \mathrm{J}_{\mathrm{p}-1}(\mathrm{x})-\mathrm{J}_{\mathrm{p}+1}(\mathrm{x})=2 \mathrm{~J}_{\mathrm{p}}^{\prime}(\mathrm{x})
\end{aligned}
$$

Term by term for $\mathrm{E}_{\mathrm{y}}^{(0)}$ :

$$
\begin{aligned}
\frac{\partial}{\partial y}\left(\frac{\partial \Pi_{x}}{\partial x}\right) & \sim-\frac{\partial}{\partial y}\left(J_{1}(u r) \frac{x}{r} u\right) \\
& \sim-\frac{\partial J_{1}}{\partial y} \frac{x}{r} u-J_{1} x \frac{\partial}{\partial y}\left(\frac{1}{r}\right) x u
\end{aligned}
$$

substituting $(\mathrm{v}=\mathrm{ur}) \quad \sim-\frac{\partial \mathrm{J}_{1}}{\partial \mathrm{v}} \frac{\mathrm{xy}}{\mathrm{r}^{2}} \mathrm{u}^{2}+\mathrm{J}_{1} \frac{\mathrm{x}}{\mathrm{r}^{2}} \frac{\mathrm{y}}{\mathrm{ur}} \mathrm{u}^{2}$

$$
\sim\left(-\frac{\partial \mathrm{J}_{1}}{\partial \mathrm{v}}+\frac{\mathrm{J}_{1}}{\mathrm{v}}\right) \frac{\mathrm{xy}}{\mathrm{r}^{2}} \mathrm{u}^{2}
$$

using (a) we get $J_{1}^{\prime}(v)=J_{0}(v)-\frac{1}{v} J_{1}(v)$ and solving for $\frac{J_{1}}{v}$ we get :

$$
\frac{\partial}{\partial y}\left(\frac{\partial \Pi_{x}}{\partial x}\right) \sim\left(-2 J_{1}^{\prime}(v)+J_{0}(v)\right) \frac{x y}{r^{2}} u^{2}
$$

now we use (b) to get $\mathrm{J}_{0}(\mathrm{v})-\mathrm{J}_{2}(\mathrm{v})=2 \mathrm{~J}_{1}^{\prime}(\mathrm{v})$ which gives:

$$
\frac{\partial}{\partial y}\left(\frac{\partial \Pi_{x}}{\partial x}\right) \sim J_{2}(v) \frac{x y}{r^{2}} u^{2} \rightarrow 0 \text { as } v \rightarrow 0
$$

For the same reason, 


$$
\frac{\partial^{2} \Pi_{z}}{\partial y \partial z} \quad \sim \quad \frac{\partial}{\partial y}\left(\frac{x}{r} J_{1}(\text { ur })\right) \rightarrow 0 \text { as } r \rightarrow 0
$$

Hence $\mathrm{E}_{\mathrm{y}}^{(0)}=0$.

For $E_{z}^{(0)}, \quad \Pi_{z} \rightarrow 0$ as $r \rightarrow 0$ and

$$
\begin{aligned}
\frac{\partial \Pi_{\mathrm{x}}}{\partial \mathrm{x}} & \sim \frac{\partial \mathrm{J}_{0}(\mathrm{ur})}{\partial(\mathrm{ur})} \frac{\partial \mathrm{r}}{\partial \mathrm{x}} \mathrm{u} \\
& \sim \frac{\partial \mathrm{J}_{0}(\mathrm{v})}{\partial \mathrm{v}} \frac{\mathrm{x}}{\mathrm{r}} \mathrm{u} \\
& \sim \quad-\mathrm{J}_{1}(\mathrm{v}) \frac{\mathrm{x}}{\mathrm{r}} \mathrm{u} \rightarrow 0 \text { as } \mathrm{r} \rightarrow 0 .
\end{aligned}
$$

Hence $E_{z}^{(0)}=0$.

Using the above method it can be shown that for a $y$ oriented dipole the $\mathrm{E}_{\mathrm{x}}^{(0)}$ and $\mathrm{E}_{\mathrm{z}}^{(0)}$ components go to 0 and for a $\mathrm{z}$ oriented dipole the $\mathrm{E}_{\mathrm{x}}^{(0)}$ and $\mathrm{E}_{\mathrm{y}}^{(0)}$ go to 0 . 


\section{APPENDIX B}

The main formulation for $d_{x x}$ and $d_{y y}$ from the MM paper is given by (notation is slightly different from MM) :

$$
\mathrm{d}_{\mu v}\left(\overrightarrow{\mathrm{k}}_{\|}, \omega \mid z z^{\prime}\right)=\sum_{\mu^{\prime}, v^{\prime}} \mathrm{g}_{\mu^{\prime} v^{\prime}}\left(\overrightarrow{\mathrm{k}}_{\|}, \omega \mid z z^{\prime}\right) \mathrm{S}_{\mu^{\prime} \mu}\left(\overrightarrow{\mathrm{k}}_{\|}\right) \mathrm{S}_{v^{\prime} v}\left(\overrightarrow{\mathrm{k}}_{\|}\right)
$$

These terms are found by solving the differential equations given in MM by:

$$
\left(\begin{array}{ccc}
\varepsilon_{0} \vec{k}^{2}-k_{y}^{2}+\frac{d^{2}}{d z^{2}} & k_{x} k_{y} & -i k_{x} \frac{d}{d z} \\
k_{x} k_{y} & \varepsilon_{0} \vec{k}^{2}-k_{x}^{2}+\frac{d^{2}}{d z^{2}} & -i k_{y} \frac{d}{d z} \\
-i k_{x} \frac{d}{d z} & -i k_{y} \frac{d}{d z} & \varepsilon_{0} \vec{k}^{2}-\vec{k}_{l}^{2}
\end{array}\right)\left(\begin{array}{ccc}
d_{x x} & d_{x y} & d_{x z} \\
d_{y x} & d_{y y} & d_{y z} \\
d_{z x} & d_{z y} & d_{z z}
\end{array}\right)=4 \pi \delta\left(z-z^{\prime}\right)\left(\begin{array}{ccc}
1 & 0 & 0 \\
0 & 1 & 0 \\
0 & 0 & 1
\end{array}\right)
$$

we can obtain the Fourier coefficients $g_{\mu \nu}$ by using the following matrix:

$S\left(\vec{k}_{\|}\right)=\frac{1}{k_{\|}}\left(\begin{array}{ccc}k_{x} & k_{y} & 0 \\ -k_{y} & k_{x} & 0 \\ 0 & 0 & k_{\|}\end{array}\right)$

to transform the vector $\left(\mathrm{k}_{\mathrm{x}}, \mathrm{k}_{\mathrm{y}}, 0\right)$ into the vector $\left(\mathrm{k}_{\|}, 0,0\right)$.

This results in the following:

$$
\left(\begin{array}{ccc}
\varepsilon_{0} \overrightarrow{\mathrm{k}}^{2}+\frac{\mathrm{d}^{2}}{\mathrm{dz} \mathrm{z}^{2}} & 0 & -\mathrm{i} \overrightarrow{\mathrm{k}}_{\|} \frac{\mathrm{d}}{\mathrm{dz}} \\
0 & \varepsilon_{0} \overrightarrow{\mathrm{k}}^{2}-\overrightarrow{\mathrm{k}}_{\|}^{2}+\frac{\mathrm{d}^{2}}{\mathrm{dz^{2 }}} & 0 \\
-\mathrm{i} \overrightarrow{\mathrm{k}}_{\|} \frac{\mathrm{d}}{\mathrm{dz}} & 0 & \varepsilon_{0} \overrightarrow{\mathrm{k}}^{2}-\overrightarrow{\mathrm{k}}_{\|}^{2}
\end{array}\right)\left(\begin{array}{lll}
\mathrm{g}_{\mathrm{xx}} & \mathrm{g}_{\mathrm{xy}} & \mathrm{g}_{\mathrm{xz}} \\
\mathrm{g}_{\mathrm{yx}} & \mathrm{g}_{\mathrm{yy}} & \mathrm{g}_{\mathrm{yz}} \\
\mathrm{g}_{\mathrm{zx}} & \mathrm{g}_{\mathrm{zy}} & \mathrm{g}_{\mathrm{zz}}
\end{array}\right)=4 \pi \delta\left(z-\mathrm{z}^{\prime}\right)\left(\begin{array}{ccc}
1 & 0 & 0 \\
0 & 1 & 0 \\
0 & 0 & 1
\end{array}\right)
$$

where $g$ is the matrix, 


$$
g=\left(\begin{array}{lll}
g_{x x} & g_{x y} & g_{x z} \\
g_{y x} & g_{y y} & g_{y z} \\
g_{z x} & g_{z y} & g_{z z}
\end{array}\right)
$$

Following MM, the dyadic becomes:

$$
\begin{aligned}
d_{x x}= & g_{x x} S_{x x} S_{x x}+g_{x y} S_{x x} S_{y x}+g_{x z} S_{x x} S_{z x} \\
& +g_{y x} S_{y x} S_{x x}+g_{y y} S_{y x} S_{y x}+g_{y z} S_{y x} S_{z x} \\
& +g_{z x} S_{z x} S_{x x}+g_{z y} S_{z x} S_{y x}+g_{z z} S_{z x} S_{z x}
\end{aligned}
$$

Collecting the non-vanishing terms leaves

$$
\begin{aligned}
& d_{x x}=g_{x x} S_{x x}^{2}+g_{y y} S_{y x}^{2} \\
& =-\frac{4 \pi c^{2}}{\omega^{2}} \frac{k_{1} k_{2}}{k_{1}-\varepsilon k_{2}} e^{i k_{2} z} \frac{k_{x}^{2}}{k_{\|}^{2}}+\frac{4 \pi i}{k_{1}-k_{2}} e^{i k_{2} z} \frac{k_{y}^{2}}{k_{\|}^{2}}
\end{aligned}
$$

where $k_{1}$ and $k_{2}$ are defined as :

$$
\begin{aligned}
& \mathrm{k}_{1}=-\left(\varepsilon \mathrm{k}^{2}-\mathrm{k}_{\|}^{2}\right)^{1 / 2} \\
& \mathrm{k}_{2}=\left\{\begin{array}{l}
\left(\mathrm{k}^{2}-\mathrm{k}_{\|}^{2}\right)^{1 / 2}, \mathrm{k}^{2}>\mathrm{k}_{\|}^{2} \\
\mathrm{i}\left(\mathrm{k}_{\|}^{2}-\mathrm{k}^{2}\right)^{1 / 2}, \mathrm{k}^{2}<\mathrm{k}_{\|}^{2} \quad ; \quad \mathrm{k}=\frac{\omega}{\mathrm{c}} \text { and } \overrightarrow{\mathrm{k}}_{\|}=\left(\mathrm{k}_{\mathrm{x}}, \mathrm{k}_{\mathrm{y}}, 0\right) .
\end{array}\right.
\end{aligned}
$$

This gives

$$
d_{x x}=\frac{4 \pi i}{k^{2} k_{\|}^{2}} e^{i k_{2} 2}\left(\frac{k^{2} k_{y}^{2}}{k_{1}-k_{2}}-\frac{k_{1} k_{2} k_{x}^{2}}{k_{1}-\varepsilon k_{2}}\right) .
$$

Similarly,

$$
\begin{aligned}
d_{y y}= & g_{x x} S_{x y} S_{x y}+g_{x y} S_{x y} S_{y y}+g_{x z} S_{x y} S_{z y} \\
& +g_{y x} S_{y y} S_{x y}+g_{y y} S_{y y} S_{y y}+g_{y z} S_{y y} S_{z y}
\end{aligned}
$$




$$
\begin{aligned}
& +g_{z x} S_{z y} S_{x y}+g_{z y} S_{z y} S_{y y}+g_{z z} S_{z y} S_{z y} \\
= & g_{x x} S_{x y}^{2}+g_{y y} S_{y y}^{2} \\
= & -\frac{4 \pi i c^{2}}{\omega^{2}} \frac{k_{1} k_{2}}{k_{1}-\varepsilon k_{2}} e^{i k_{2} z} \frac{k_{y}^{2}}{k_{l}^{2}}+\frac{4 \pi i}{k_{1}-k_{2}} e^{i k_{2} z} \frac{k_{x}^{2}}{k_{l}^{2}}
\end{aligned}
$$

giving $d_{y y}$ as,

$$
d_{y y}=\frac{4 \pi i}{k^{2} k_{l}^{2}} e^{i k_{2} z}\left(\frac{k^{2} k_{x}^{2}}{k_{1}-k_{2}}-\frac{k_{1} k_{2} k_{y}^{2}}{k_{1}-\varepsilon k_{2}}\right)
$$




\section{APPENDIX C}

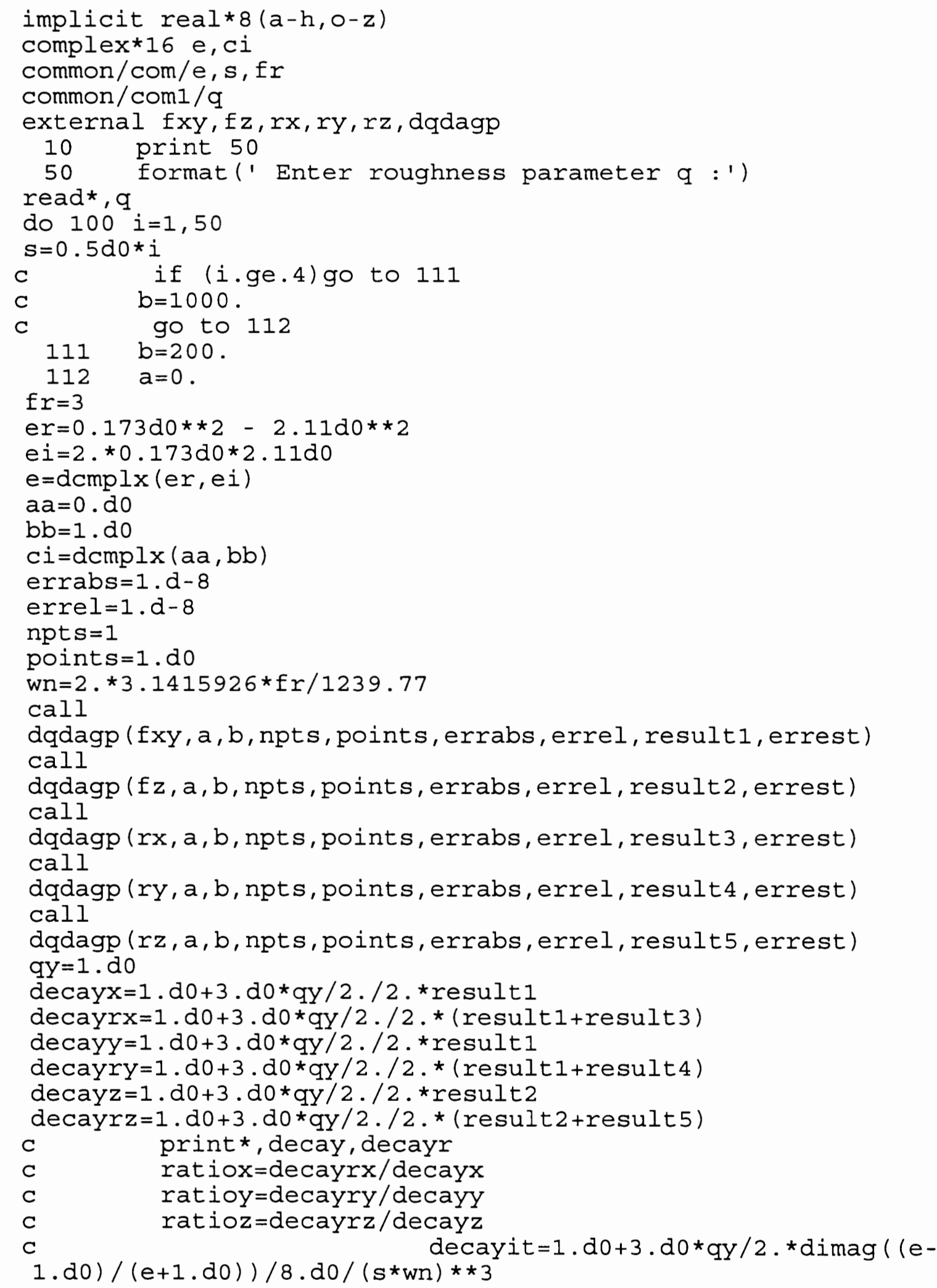




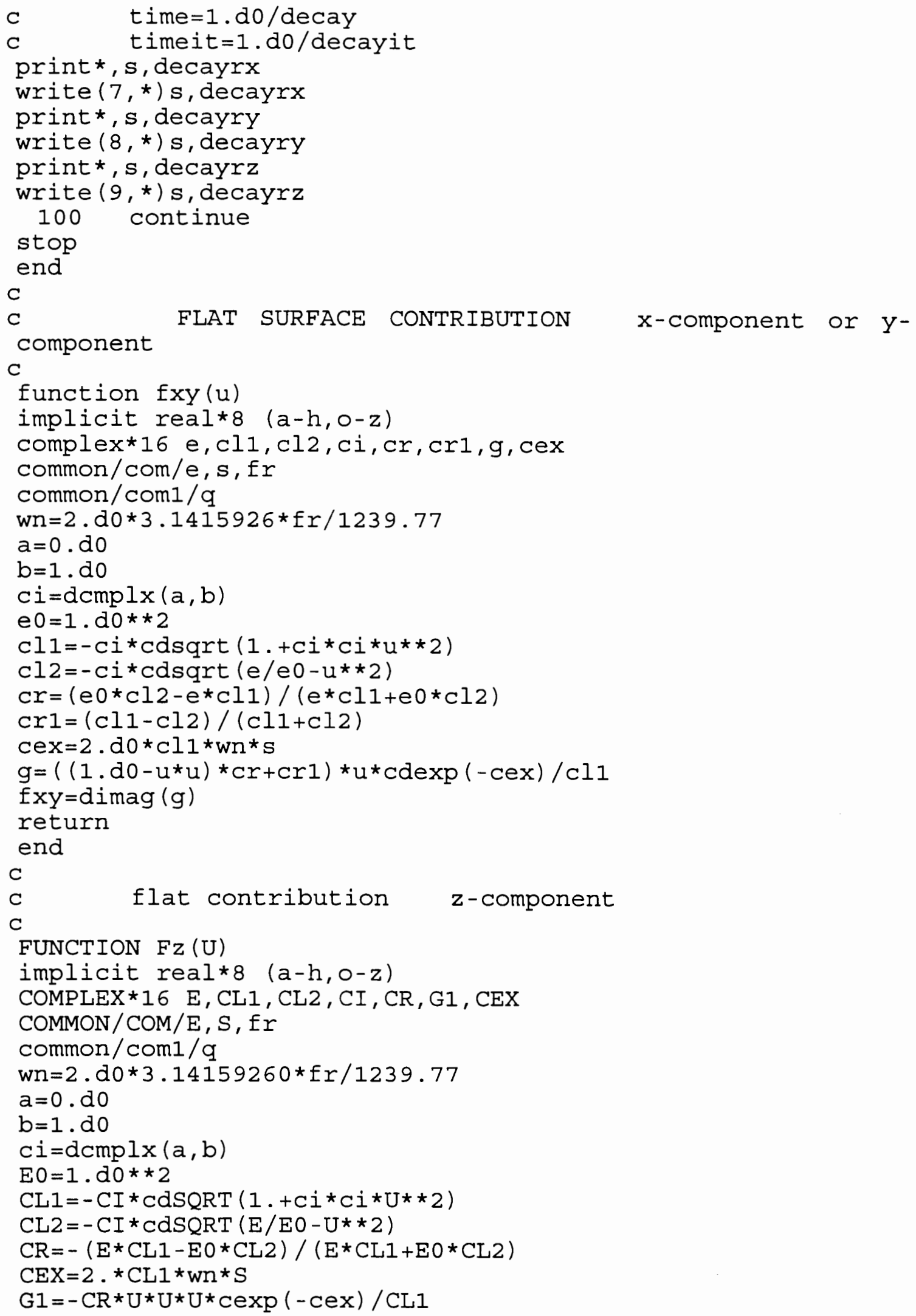


$f z=\operatorname{dimag}(g 1)$

return

end

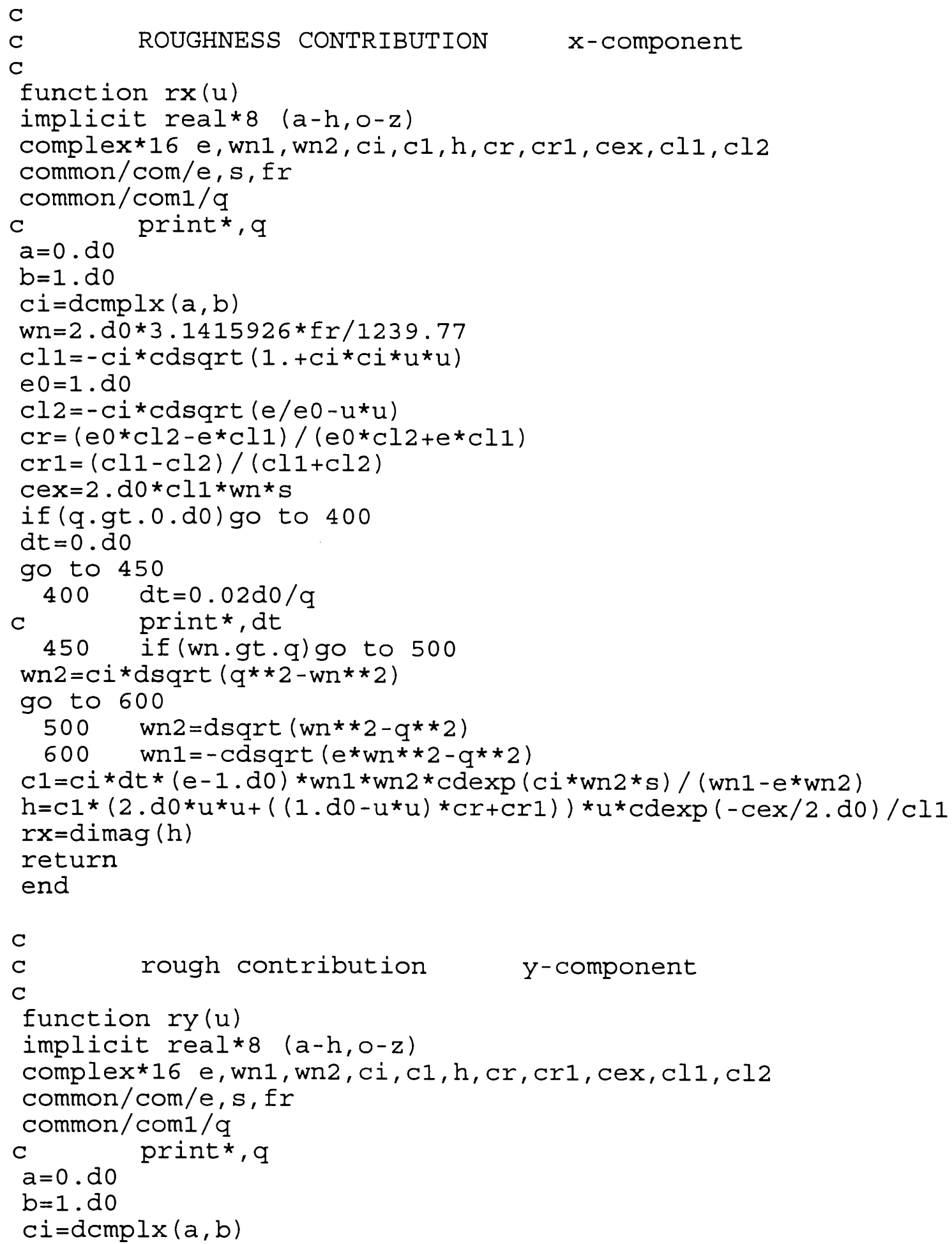




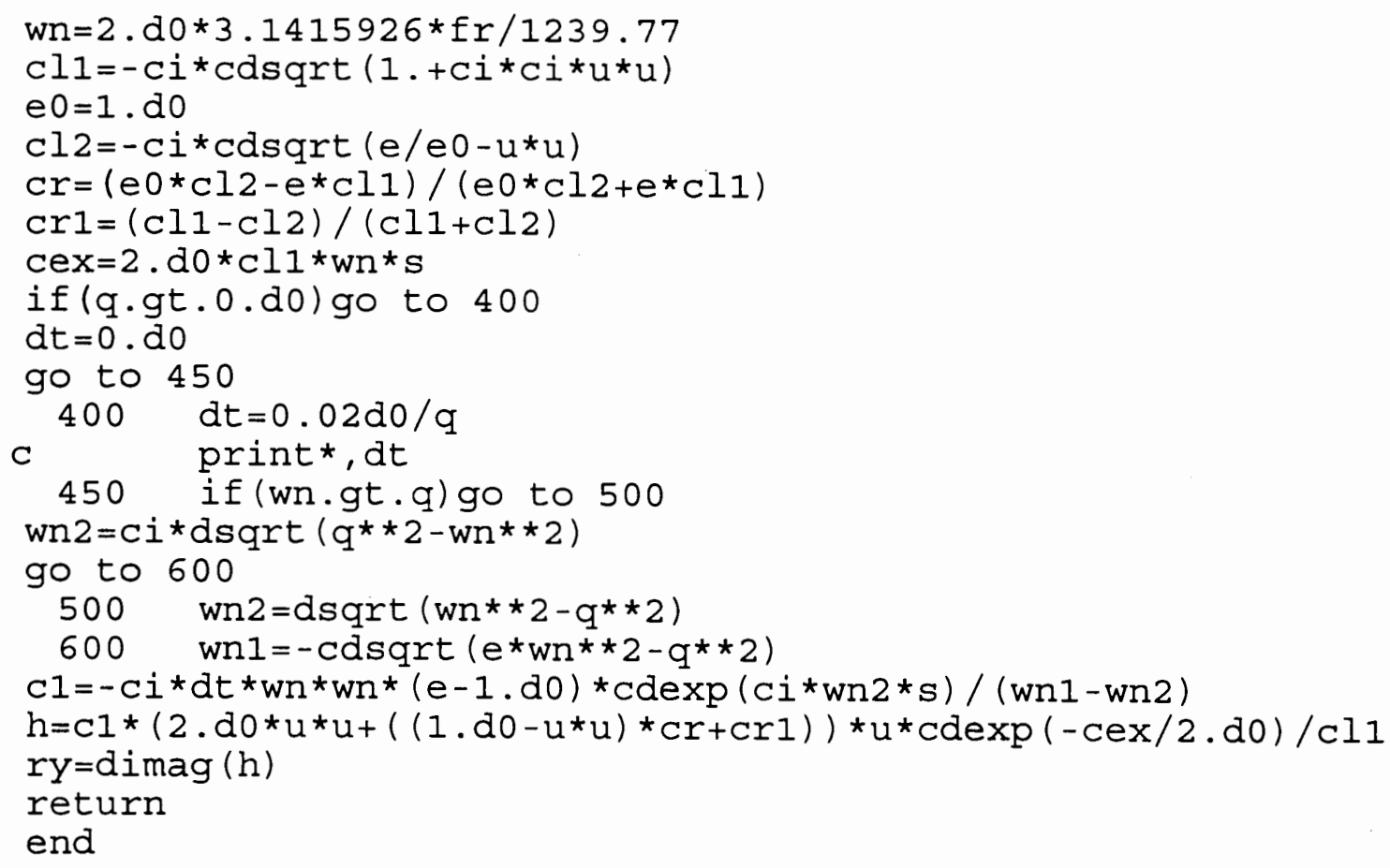

C

C rough contribution z-component

C

FUNCTION rz(U)

implicit real*8 $(a-h, o-z)$

COMPLEX *16 E, CL1, CL2, CI, CR, G2, CEX, NUM, DEN, ANS, wk1, WK2

COMMON/COM/E, S, fr

common/coml/q

$w n=2 . d 0 * 3.14159260 * f r / 1239.77$

$a=0 . d 0$

$\mathrm{b}=1 . \mathrm{d} 0$

ci $=$ dcmplx $(a, b)$

$\mathrm{EO}=1 . \mathrm{dO} * * 2$

$\mathrm{CLI}=-\mathrm{CI} * \mathrm{CdSQRT}(1+\mathrm{Ci} * \mathrm{Ci} * \mathrm{U} * \star 2)$

$\mathrm{CL} 2=-\mathrm{CI} * \mathrm{CdSQRT}(\mathrm{E} / \mathrm{E} 0-\mathrm{U} * \star 2)$

$\mathrm{CR}=-(\mathrm{E} * \mathrm{CL} 1-\mathrm{E} 0 * \mathrm{CL} 2) /(\mathrm{E} * \mathrm{CL} 1+\mathrm{E} 0 * \mathrm{CL} 2)$

$\mathrm{CEX}=2 . * \mathrm{CL} 1 * W n * \mathrm{~S}$

$W K 1=-\operatorname{cdsqrt}(E * W N * \star 2-Q * \star 2)$

if $(q . g t .0 . d 0)$ go to 400

$d t=0 . d 0$

go to 450

$400 \quad \mathrm{dt}=0.02 \mathrm{~d} 0 / \mathrm{q}$

c print*,dt

450 if (wn.gt.q) go to 500

$\mathrm{WK} 2=\mathrm{CI} *(Q * \star 2-\mathrm{WN} * \star 2) \star \star .5$

go to 600

$500 \quad W K 2=-C i * C i *(W N * \star 2-Q * * 2) * * .5$

$600 \quad \mathrm{NUM}=\mathrm{CI} * \mathrm{dt} *(\mathrm{E}-1) * \mathrm{Q} * \mathrm{Q} * \operatorname{CdEXP}(\mathrm{CI} * \mathrm{WK} 2 * \mathrm{~S})$ 
$\mathrm{DEN}=\mathrm{WK} 1-\mathrm{E} * \mathrm{WK} 2$

ANS $=$ NUM $/ D E N$

$\mathrm{G} 2=-\mathrm{ANS} *(1 .-\mathrm{CR}) * \mathrm{U} * \mathrm{U} * \mathrm{U} * \mathrm{CdEXP}(-\mathrm{CL} 1 * \mathrm{WN} * \mathrm{~S}) / \mathrm{CL} 1$

$r z=D I M A G(G 2)$

RETURN

END 